\section{Bile acids regulate intestinal antigen presentation and reduce graft-versus-host disease without impairing the graft-versus-leukemia effect}

\author{
Eileen Haring, ${ }^{1,2^{*}}$ Franziska M. Uhl, ${ }^{1,2 *}$ Geoffroy Andrieux, ${ }^{3,4}$ Michele Proietti, ${ }^{5}$ \\ Alla Bulashevska, ${ }^{5}$ Barbara Sauer, ${ }^{1}$ Lukas M. Braun, ${ }^{1,2}$ \\ Enrique de Vega Gomez, ${ }^{1}$ Philipp R. Esser, ${ }^{6}$ Stefan F. Martin, ${ }^{6}$ Dietmar Pfeifer, ${ }^{1}$ \\ Marie Follo, ${ }^{1}$ Annette Schmitt-Graeff, ${ }^{7}$ Joerg Buescher, ${ }^{8}$ Justus Duyster, ${ }^{1}$ \\ Bodo Grimbacher, ${ }^{5,9,10,11}$ Melanie Boerries, ${ }^{3,4,12}$ Erika L. Pearce, ${ }^{8}$ \\ Robert Zeiser ${ }^{1,4,9}$ and Petya Apostolova ${ }^{1}$
}

${ }^{1}$ Department of Medicine I, Medical Center - University of Freiburg, Faculty of Medicine, University of Freiburg; ${ }^{2}$ Faculty of Biology, Albert-Ludwigs-University, Freiburg; ${ }^{3}$ Institute of Medical Bioinformatics and Systems Medicine, Medical Center - University of Freiburg, Faculty of Medicine, University of Freiburg, Freiburg; ${ }^{4}$ German Cancer Consortium (DKTK), partner site Freiburg; and German Cancer Research Center (DKFZ), Heidelberg; ${ }^{5}$ Institute for Immunodeficiency, Center for Chronic Immunodeficiency (CCI), Medical Center, Faculty of Medicine, Albert-Ludwigs-University, Freiburg; ${ }^{6}$ Allergy Research Group, Department of Dermatology, Medical Center - University of Freiburg, Faculty of Medicine, University of Freiburg; ${ }^{7}$ University of Freiburg; ${ }^{8}$ Max-Planck Institute of Immunobiology and Epigenetics, Freiburg; ${ }^{9} \mathrm{CIBSS}$ - Centre for Integrative Biological Signalling Studies, University of Freiburg, Freiburg; ${ }^{10}$ DZIF - German Center for Infection Research, Satellite Center Freiburg; ${ }^{11}$ RESIST - Cluster of Excellence 2155 to Hanover Medical School, Satellite Center Freiburg and ${ }^{12}$ Comprehensive Cancer Centre Freiburg (CCCF), University Medical Center Freiburg, Faculty of Medicine, University of Freiburg, Freiburg, Germany

*EH and FMU contributed equally as co-first authors.

\section{ABSTRACT}

A cute graft-versus-host disease (GvHD) causes significant mortality in patients undergoing allogeneic hematopoietic cell transplantation. Immunosuppressive treatment for GvHD can impair the beneficial graft-versus-leukemia effect and facilitate malignancy relapse. Therefore, novel approaches that protect and regenerate injured tissues without impeding the donor immune system are needed. Bile acids regulate multiple cellular processes and are in close contact with the intestinal epithelium, a major target of acute GvHD. Here, we found that the bile acid pool is reduced following GvHD induction in a preclinical model. We evaluated the efficacy of bile acids to protect the intestinal epithelium without reducing anti-tumor immunity. We observed that application of bile acids decreased cytokine-induced cell death in intestinal organoids and cell lines. Systemic prophylactic administration of tauroursodeoxycholic acid (TUDCA), the most potent compound in our in vitro studies, reduced GvHD severity in three different murine transplantation models. This effect was mediated by decreased activity of the antigen presentation machinery and subsequent prevention of apoptosis of the intestinal epithelium. Moreover, bile acid administration did not alter the bacterial composition in the intestine suggesting that its effects are cell-specific and independent of the microbiome. Treatment of human and murine leukemic cell lines with TUDCA did not interfere with the expression of antigen presentation-related molecules. Systemic T-cell expansion and especially their cytotoxic capacity against leukemic cells remained intact. This study establishes a role for bile acids in the prevention of acute GvHD without impairing the graft-versus-leukemia effect. In particular, we provide a scientific rationale for the systematic use of TUDCA in patients undergoing allogeneic hematopoietic cell transplantation.
Haematologica 2021

Volume 106(8):2131-2146

\section{Correspondence:}

PETYA APOSTOLOVA

petya.apostolova@uniklinik-freiburg.de

Received: November 15, 2019.

Accepted: July 7, 2020.

Pre-published: July 16, 2020.

https://doi.org/10.3324/haematol.2019.242990

(C)2021 Ferrata Storti Foundation

Material published in Haematologica is covered by copyright. All rights are reserved to the Ferrata Storti Foundation. Use of published material is allowed under the following terms and conditions:

https://creativecommons.org/licenses/by-nc/4.0/legalcode. Copies of published material are allowed for personal or internal use. Sharing published material for non-commercial purposes is subject to the following conditions:

https://creativecommons.org/licenses/by-nc/4.0/legalcode, sect. 3. Reproducing and sharing published material for commercial purposes is not allowed without permission in writing from the publisher. 


\section{Introduction}

Allogeneic hematopoietic cell transplantation (allo-HCT) is a potentially curative treatment for leukemia and lymphoma. However, approximately $50 \%$ of acute myeloid leukemia patients develop a malignancy relapse after alloHCT. ${ }^{1}$ One contributing factor is the administration of immunosuppression as a prophylaxis or treatment for acute graft-versus-host disease (aGvHD). aGvHD is a severe transplant-associated complication which affects half of all alloHCT recipients. ${ }^{2}$ Compared to other clinical manifestations of aGvHD, intestinal GvHD causes the highest mortality, especially if it is refractory to corticosteroid treatment. ${ }^{3}$ The pathogenesis of aGvHD is marked by activation of donor $\mathrm{T}$ cells and their expansion and migration to the target organs liver, skin and gastrointestinal tract where they cause tissue damage either by direct cytotoxicity or via cytokine secretion. A hallmark of intestinal aGvHD is the damage of intestinal stem cells (ISC) and Paneth cells. ${ }^{4,5}$ Recent work has shown that interferon $\gamma$ (IFN $\gamma$ ), a cytokine commonly increased in $\mathrm{aGvHD}$, is responsible for intestinal tissue damage. ${ }^{6,7}$ Current approved GvHD prophylaxis strategies are based on immunosuppressive drugs that deplete $\mathrm{T}$ cells or modulate their activity and cytokine signaling. Novel developments aiming at regenerating the intestinal epithelium would allow a reduction of broad-spectrum immunosuppression and potentially decrease the relapse rates after allo-HCT.

There are two main classes of bile acids. Primary bile acids are generated in the liver via cholesterol catabolism. In the final step of their synthesis, they are conjugated to the amino acids glycine and taurine. This step stabilizes their amphipathic structure that is necessary to keep their full emulsifying activity in the intestine. After fulfilling their digestive role, most bile acids (appoximately 90-95\%) are actively taken up by the intestinal epithelial cells and transported back to the liver. ${ }^{8}$ Primary bile acids, which are not re-absorbed, can be converted into secondary bile acids via modification and metabolization by microbial enzymatic activity thereby influencing the microbiome composition. ${ }^{9}$ Bile acids have cytoprotective and anti-apoptotic properties for hepatocytes ${ }^{10,11}$ via the preservation of mitochondrial glutathione ${ }^{12}$ Moreover, they modulate the translocation of apoptosis-regulating proteins $\mathrm{BCL}-2$-associated $\mathrm{X}$ protein (BAX) and BCL-2-antagonist-of-cell-death (BAD) from the cytosol to the mitochondria and vice versa. ${ }^{13,14}$ Also immunomodulatory effects of bile acids and their receptors have been described. The two most prominent bile acid receptors are the Farnesoid X receptor (FXR) and the transmembrane G protein-coupled bile acid receptor 5 (TGR5). FXR is expressed in both liver and intestine and was shown that mice lacking FXR were more prone to development of acute and chronic colitis. ${ }^{15}$ Intestinal damage during GvHD has been previously linked with bile acid malabsorption. ${ }^{16}$ In a recent study, metabolomic analysis of serum samples from allo-HCT recipients showed alterations in bile acid levels among other metabolites. ${ }^{17}$ So far, no data on bile acid levels in liver, intestines or in intestinal content in the context of GvHD have been available.

The aim of this study was to investigate whether alloHCT causes alterations in the bile acid composition and whether exogenous bile acid application can positively influence aGvHD development. We show that the bile acid pool was depleted after allo-HCT. Administration of tauroursodeoxycholic acid (TUDCA) improved aGvHD out- come by direct protection of the intestinal epithelium from cytokine damage and by lowering antigen presentation by non-hematopoietic cells. This makes it an attractive compound for combination therapy with already clinically available immunosuppressive strategies.

\section{Methods}

\section{Mice}

$\mathrm{BALB} / \mathrm{c}\left(\mathrm{H}-2 \mathrm{~K}^{\mathrm{d}}\right)$ and $\mathrm{C} 57 \mathrm{BL} / 6\left(\mathrm{H}-2 \mathrm{~K}^{\mathrm{b}}\right)$ mice were purchased from the animal facility at Freiburg University Medical Center or from Janvier Labs (Le Genest-Saint-Isle, France). Luciferase-transgenic C57BL/6 mice $\left(\mathrm{H}-2 \mathrm{~K}^{b}\right)$ were bred in the animal facility of the Center for Clinical Research at Freiburg University Medical Center (Freiburg i.Br., Germany). B6.129P2-Lgr $5^{\mathrm{tm1}(\mathrm{cre} / \mathrm{RRT2}) \mathrm{Cle} / \mathrm{I}}$ mice on the C57BL/6 background $\left(\mathrm{H}-2 \mathrm{~K}^{b}\right)$ were a kind gift from Prof. Roland Schüle (Center for Clinical Research, Freiburg i.Br.). All animals were housed under specific pathogen-free conditions at the animal facility of the Center for Clinical Research (ZKF, Freiburg i.Br., Germany). All animal protocols (G-13/045, G-16/018, G-17/063; X-13/07J; X-15/10A) were approved by the Federal Ministry for Nature, Environment and Consumer Protection of the state of Baden-Württemberg, Germany.

All other materials and methods are provided in the Online Supplementary Appendix.

\section{Results}

\section{Allogeneic hematopoietic cell transplantation alters the levels of bile acids and the expression of bile acid receptors}

We first investigated how allo-HCT influences the enterohepatic circulation (Figure 1A) and the bile acid pool. We extracted metabolites from liver, ileum, ileal contents and serum of untreated mice and mice developing aGvHD (Figure 1B). We measured the levels of eight major bile acid metabolites by liquid chromatography - mass spectrometry (LC-MS) (Figure 1C) and observed that taurocholic acid (TCA) was the predominant bile acid in all four compartments. Induction of aGvHD resulted in reduced bile acid pools. While the levels of most bile acids significantly decreased, the absolute amount of ursodeoxycholic acid (UDCA) increased (Figure 1D; Online Supplementary Figure S1A to C).

Another important determinant of bile acid signaling is the expression of bile acid receptors. We found that intestinal Nr1h4 mRNA levels (encoding for FXR) increased and Gpbar1 levels (encoding for TGR5) decreased with a peak on day 4 after bone marrow transplantation (BMT) (Figure 1E; Online Supplementary Figure S1D). Collectively, our results show that allo-HCT causes a reduction of the bile acid pool in liver, intestine and intestinal content as well as a deviation in bile acid receptor expression.

\section{Treatment with bile acids prevents cell death of intestinal organoids and cell lines}

Small intestinal organoids mimic the structure of the intestine. ${ }^{18}$ We used this in vitro system to test the impact of different bile acids on the viability and morphology of the intestinal epithelium. We selected bile acids belonging to different groups for our analysis: chenodeoxycholic acid (CDCA) as a primary bile acid, UDCA as a secondary unconjugated bile acid, TUDCA as a secondary conjugated 
A

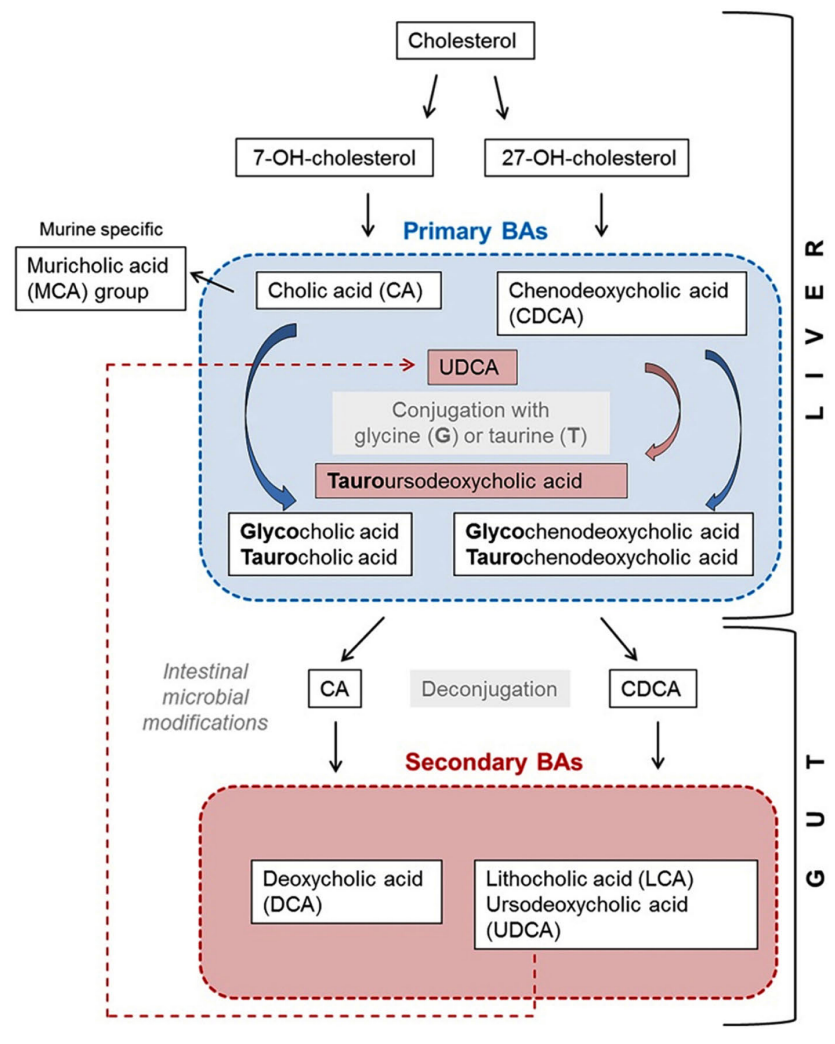

B
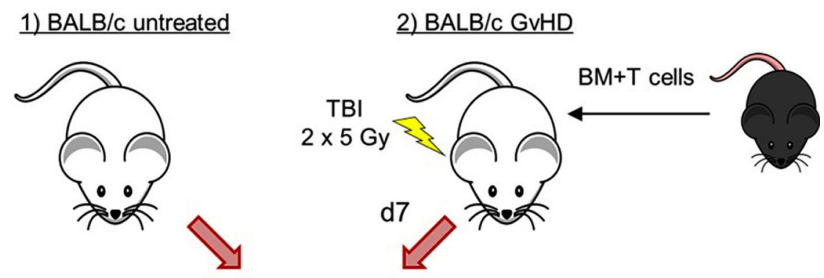

Metabolite extraction from serum, liver, ileum and ileal contents

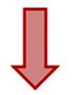

C

\section{Analysis via LC-MS}

\begin{tabular}{|l|l|l|}
\hline \multirow{4}{*}{$\begin{array}{l}\text { Unconjugated } \\
\text { primary bile acids }\end{array}$} & MCA & Muricholic acid \\
\cline { 2 - 3 } & CA & Cholic acid \\
\cline { 2 - 3 } & CDCA & Chenodeoxycholic acid \\
\hline \multirow{3}{*}{$\begin{array}{l}\text { Conjugated primary } \\
\text { bile acids }\end{array}$} & GCA & Glycocholic acid \\
\cline { 2 - 3 } & TCA & Taurocholic acid \\
\cline { 2 - 3 } & TCDCA & Taurochenodeoxycholic acid \\
\hline \multirow{2}{*}{$\begin{array}{l}\text { Secondary bile acids } \\
\text { and conjugates }\end{array}$} & UDCA & Ursodeoxycholic acid \\
\cline { 2 - 3 } & TUDCA & Tauroursodeoxycholic acid \\
\hline
\end{tabular}

Ileal contents
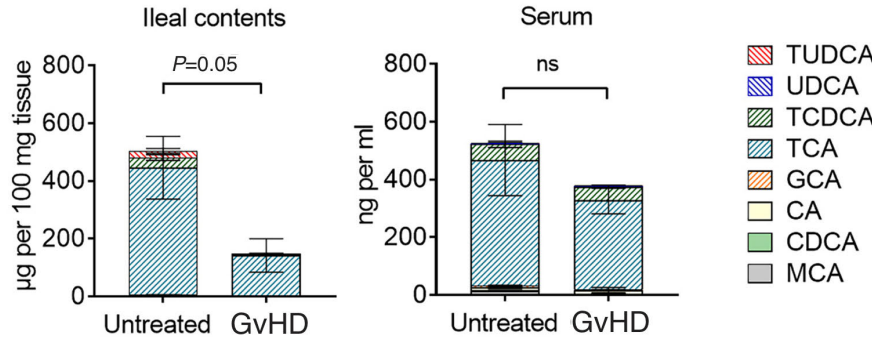

Nr1h4/Gpbar1

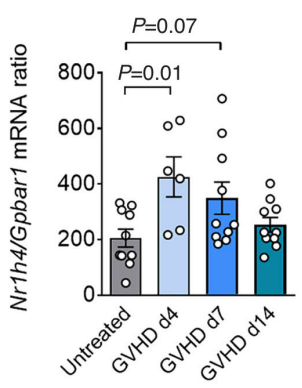

Figure 1. Allogeneic hematopoietic cell transplantation induces changes in bile acid levels and bile acid receptor expression. (A) Simplified schematic overview of bile acid metabolism and the enterohepatic circulation. (B) Experimental flow for metabolomic profiling of the bile acid pool from untreated BALB/c mice and mice developing graft-versus-host disease (GvHD). (C) Overview of the bile acids quantified by liquid chromatography - mass spectrometry. (D) Quantification of the bile acid composition in liver, ileum, ileal contents and serum of untreated mice and on day 7 after bone marrow transplantation (BMT). Data were pooled from five individual mice per group and are presented as mean \pm standard error of the mean for each metabolite. $P$-values were calculated for the total bile acid pool using the two-tailed unpaired Student's $t$-test; ns: not significant. (E) Quantitative real-time polymerase chain reaction analysis of the mRNA expression of the bile acid receptors Nr1h4 (encodes for the FXR protein) and Gpbar1 (encodes for the TGR5 protein) in the small intestine of untreated mice and mice developing GvHD. Data are pooled from $n=10$ mice in the untreated, GvHD d7 and GvHD d14 groups and $n=5$ mice in the GvHD day 4 group. The $P$-values were calculated using the ordinary one-way ANOVA test with correction for multiple comparisons. 
A

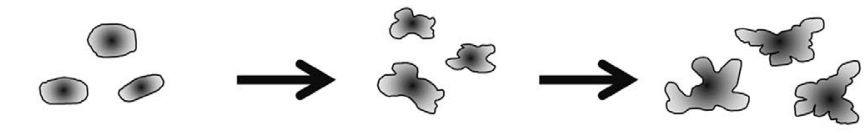

d0: split organoids $+\quad$ d3: medium change $+\quad$ d6: read-outs

bile acid/vehicle bile acid/vehicle +

B

TNF / IFNY

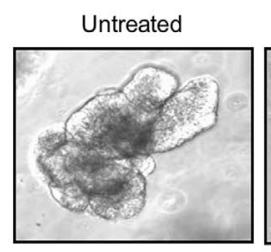

TNF+vehicle
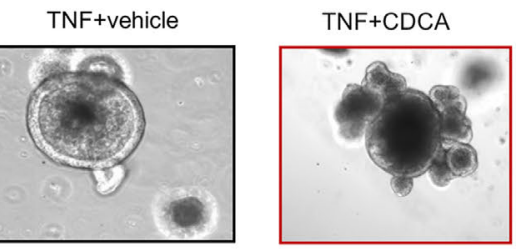

TNF+UDCA

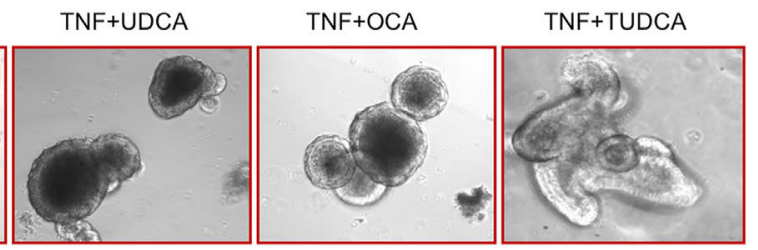

TNF+TUDCA

C

D
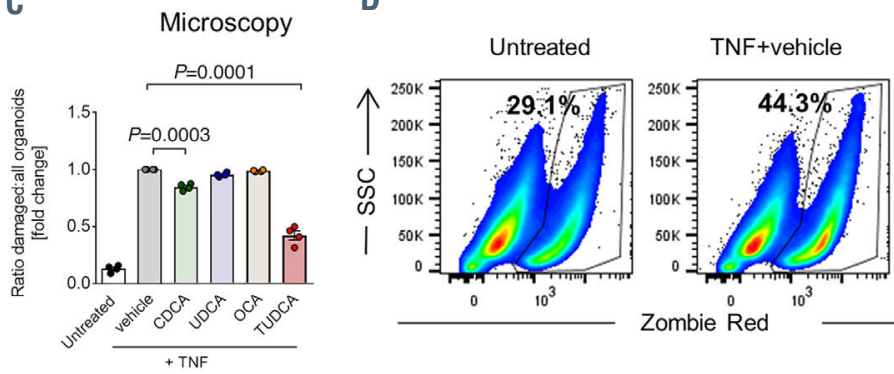

TNF+TUDCA

E

F

Untreated

$\mathrm{IFN} \gamma+$ +vehicle

IFNy+CDCA

IFNy+UDCA
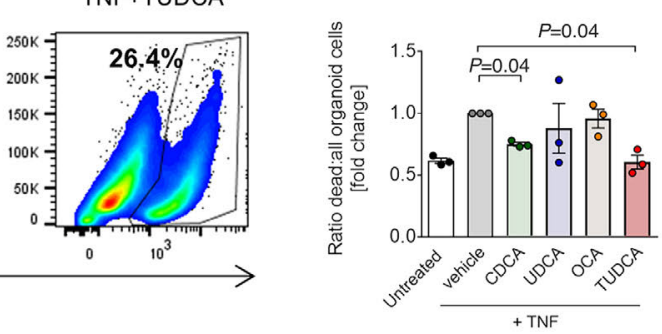

Flow cytometry
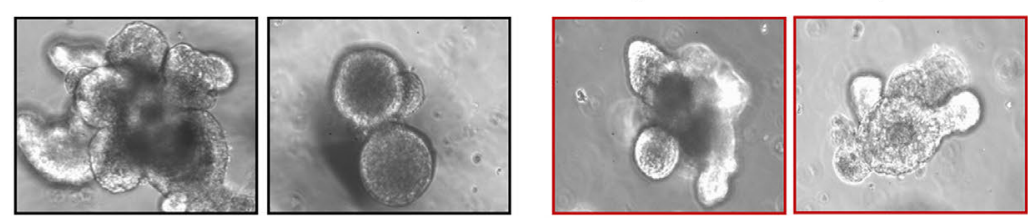

IFNy+OCA

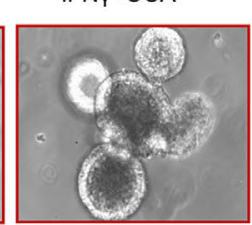

IFNy+TUDCA

G

Microscopy

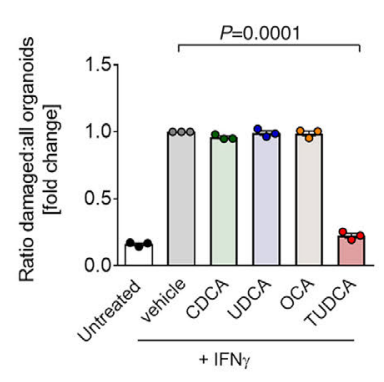

TUDCA

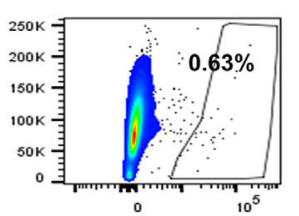

Zombie Red
H

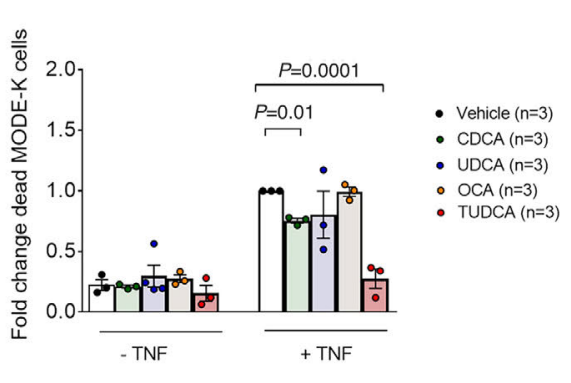

Figure 2. Treatment with bile acids reduces cell death in small intestinal organoids and MODE-K cells. (A) Experimental setting for the treatment of organoids with tumor necrosis factor (TNF) $(20 \mathrm{ng} / \mathrm{mL})$, interferon $\gamma(\mathrm{IFN} \gamma)(2.5 \mathrm{ng} / \mathrm{mL})$ and the respective bile acids. (B) Representative images of BALB/c intestinal organoids treated with TNF as described in (A). (C) Quantification of damaged organoids treated with TNF as in (A) performed by manual microscope counting. Data were normalized to the TNF plus (TNF+)vehicle group. Statistical analysis of $n=4$ biologically independent experiments. The $P$-value was calculated using an ordinary one-way ANOVA test with correction for multiple comparisons. (D and E) Intestinal organoids were cultured as described in (A). Organoids were digested and the proportion of dead cells was determined by flow cytometry. (D) Representative flow cytometry dot plots. (E) Quantification of the percentages of dead cells. Data were normalized to the TNF+vehicle group. Statistical analysis of $n=3$ biologically independent experiments. The $P$-value was calculated using the ordinary one-way ANOVA test with correction for multiple comparisons. (F) Representative images of BALB/C intestinal organoids treated with IFNy as described in (A). (G) Quantification of damaged organoids treated with IFN $\gamma$ as in (A) performed by manual microscope counting. Data were normalized to the IFN $\gamma+v e h i c l e$ group. Statistical analysis of $n=3$ biologically independent experiments. The $P$-value was calculated using an ordinary one-way ANOVA test with correction for multiple comparisons. (H and I) Analysis of MODE-K cell viability after treatment with TNF \pm chenodeoxycholic acid (CDCA), ursodeoxycholic acid (UDCA), 6-ethylchenodeoxycholic acid (OCA) or tauroursodeoxycholic acid (TUDCA) for 48 hours performed by flow cytometry. $(\mathrm{H})$ Quantification of the percentages of dead cells. Data were normalized to the TNF+vehicle group. Statistical analysis of $n=3$ biologically independent experiments performed in technical duplicates or triplicates. The $P$-values were calculated using the ordinary oneway ANOVA test with correction for multiple comparisons. (I) Representative flow cytometry dot plots. 
bile acid and 6-ethylchenodeoxycholic acid (obeticholic acid, OCA) as a synthetic compound and the strongest agonist of the FXR receptor. We evaluated the toxicity of these bile acids on organoids and the intestinal cell line MODE-K to select non-toxic doses for further experiments (Online Supplementary Figure S2A and B). Organoids were treated with tumor necrosis factor (TNF) or IFN $\gamma$ in combination with or without bile acids (Figure 2A). We observed that TNF treatment, performed to induce cellular damage, reduced organoid viability as shown by changes in cell morphology and organoid structure. This effect was partially reversed by addition of CDCA and more significantly of TUDCA (Figure 2B to E). We confirmed the beneficial effect of TUDCA on organoid viability using IFN $\gamma$ to induce organoid damage (Figure 2F and G). As TUDCA was the most potent bile acid to protect intestinal organoids from cytokine-induced cell death, we performed quantitative polymerase chain reaction (qPCR) analysis from organoids treated with TNF \pm TUDCA in order to evaluate which cell types were particularly preserved. We observed a strong upregulation of the anti-microbial peptides defensin $\alpha 1$ and 4 (Defa1 and Defa4) which are produced by Paneth cells (Online Supplementary Figure S2C). Additionally, we identified an elevated expression of the intestinal stem cell (ISC) marker leucine-rich repeat-containing G-protein coupled receptor 5 (Lgr5). Although there was a trend towards an increase in the goblet cell marker mucin 2 (Muc2) and the enteroendocrine cell marker chromogranine A (Chga), these changes were not significant (Online Supplementary Figure S2C). We confirmed the hypothesis that bile acids protect intestinal cells from cell death via performing additional experiments using the murine small intestine cell line MODE-K. TNF treatment reduced the viability of MODE$\mathrm{K}$ cells. This was reversed partly by CDCA and even more by TUDCA (Figure $2 \mathrm{H}$ and I). Together, these data indicate that exposure to bile acids reduces intestinal cell damage induced by pro-inflammatory cytokines.

\section{Prophylactic administration of bile acids reduces acute graft-versus-host disease severity in mice through a cell-intrinsic effect independent of the microbiome}

Our in vitro studies provided evidence that bile acids protect the intestinal epithelium. Since TUDCA showed the strongest effects, we used this compound for further studies in a murine in vivo BMT model (Figure $3 \mathrm{~A}$ and $\mathrm{C}$ ). We determined bile acid concentrations and confirmed an increase of TUDCA levels after exogenous administration. Levels increased from $0.5 \%$ to $35 \%$ of the measured bile acids in the serum and from $3.2 \%$ to $57 \%$ in the ileal content reaching a concentration of almost $1 \mathrm{mg}$ per $100 \mathrm{mg}$ (Online Supplementary Figure S3A and B). Survival was significantly improved in animals treated with TUDCA in two different transplantation models using total body irradiation (TBI) as the conditioning treatment (Figure $3 \mathrm{~B}$ and $\mathrm{D}$ ). As a confirmation, histopathological analysis of the liver, small intestine and colon of recipient mice demonstrated significantly reduced aGvHD scores in the TUDCA-treated group (Figure 3E). This was further validated in an irradiation-free model with chemotherapy conditioning (Online Supplementary Figure $S 3 C$ and D). Pro-inflammatory cytokines contribute to tissue damage and T-cell activation during aGvHD. We observed reduced serum cytokine levels in the treatment group with significant differences for TNF and a trend towards lower levels of IFN (Figure 3F).
These data demonstrate a protective effect of TUDCA in the prophylactic setting with improved survival rates, reduced histological aGvHD scores and lower concentrations of pro-inflammatory cytokines.

Since we found in our in vitro system an effect on intestinal cell viability by other bile acids as well, we assessed the impact of CDCA and UDCA as a prophylaxis for aGvHD (Online Supplementary Figure S3E). Histopathological analysis revealed a slight improvement upon CDCA administration (Online Supplementary Figure $S 3 F$ ) and a more pronounced effect after treatment with UDCA (Online Supplementary Figure S3G).

Intestinal bacteria play an essential role in bile acid metabolism. They generate secondary bile acids by removing glycine or taurine residues from primary bile acids, which were not reabsorbed from intestinal epithelial cells and recycled in the enterohepatic circle. ${ }^{19,20}$ The microbiome composition and the bile acid pool influence each other. ${ }^{21}$ Recent data show that aGvHD is associated with changes in the microbiome including a diminished microbial diversity. ${ }^{22-24}$ We therefore investigated whether TUDCA potentially mediates its protective effects by acting directly on the intestinal cells or by modifying the intestinal microbiome composition. Microbiome analysis was conducted in mice developing aGvHD. We could detect the expected reduction in microbial diversity in both groups compared to untreated mice. This reduction was independent of TUDCA treatment as depicted by similar Shannon indices and inversed Simpson indices (Figure 3G and H). Detailed analysis of the relative abundance of different species confirmed that the microbiome is altered during aGvHD development. In line with previous reports, Lactobacillus numbers increased and certain anaerobic species (in our case Muribaculum and Sporobacter) decreased. TUDCA- and vehicle-treated mice exhibited similar relative abundance profiles suggesting that TUDCA protects the intestinal epithelium by directly affecting the intestinal cells and not by changing the microbial composition (Figure 3I). Supporting this concept, TUDCA preserved its beneficial effects on the survival of mice developing aGvHD even after decontaminating antibiotic treatment that was administered prior to BMT (Figure 3J and $\mathrm{K}$ ). Of note, antibiotic treatment did not lead to the general reduction in the bile acid pool of the ileal content seen as a result from GvHD induction (Online Supplementary Figure SH3). Together, these data suggest that although bile acids and the microbiome are closely connected in the intestinal tract, the beneficial effect of TUDCA on GvHD outcome is not mainly mediated by regulation of the intestinal bacteria.

\section{Bile acid treatment decreases intestinal antigen presentation}

In order to better understand the mechanism by which bile acid administration protects the intestine from aGvHDinduced damage, we performed gene expression analysis of small intestinal samples on day 14 after BMT. Using samples from TUDCA-treated animals and controls, we performed a hypergeometric test of significant regulated genes ( $q$-value $<0.05$ ) to find differentially regulated terms from the Gene Ontology database. The most significantly downregulated pathways upon TUDCA treatment included pathways related to immune activation, among others the gene set 'antigen processing and presentation' (Figure 4A). Expression analysis in a second independent cohort confirmed the data on the mRNA and protein level (Figure 4B 
A

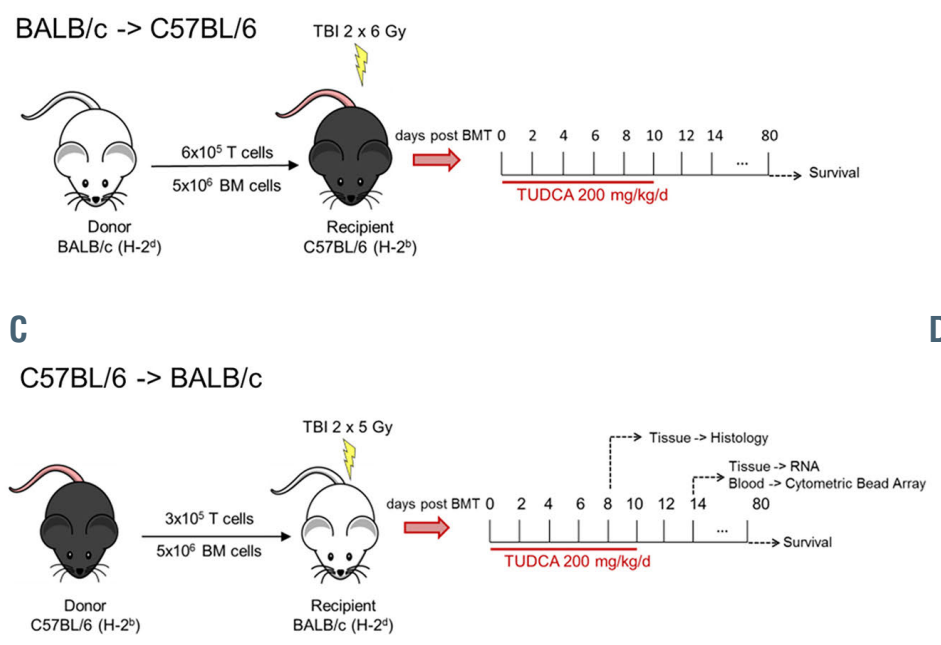

B

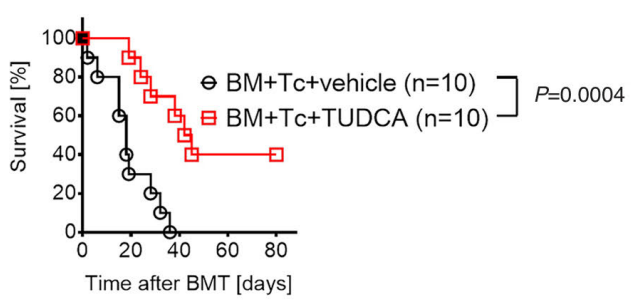

D

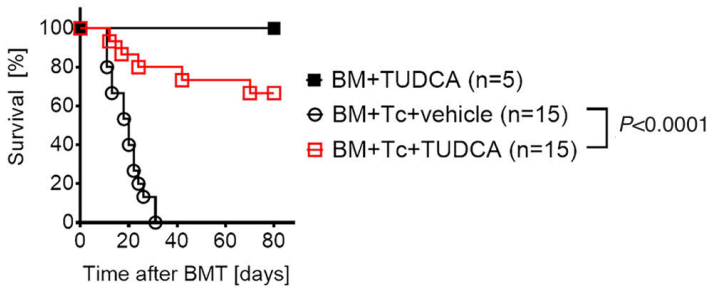

E

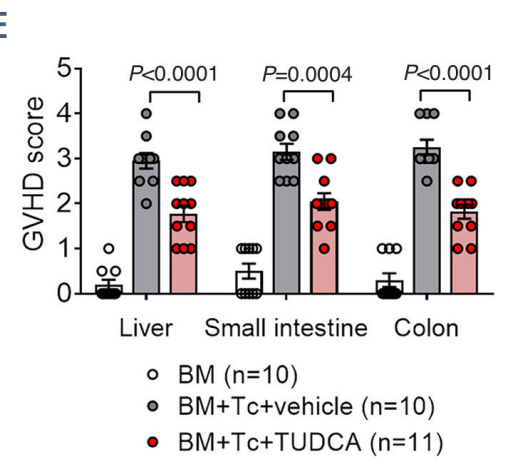

G

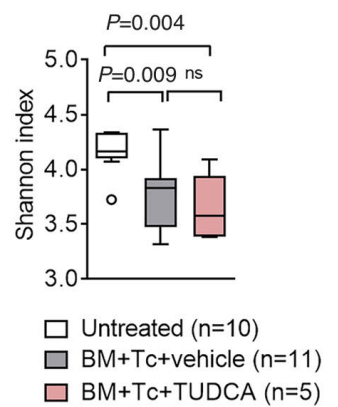

H

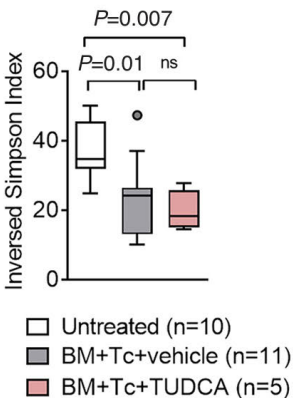

J

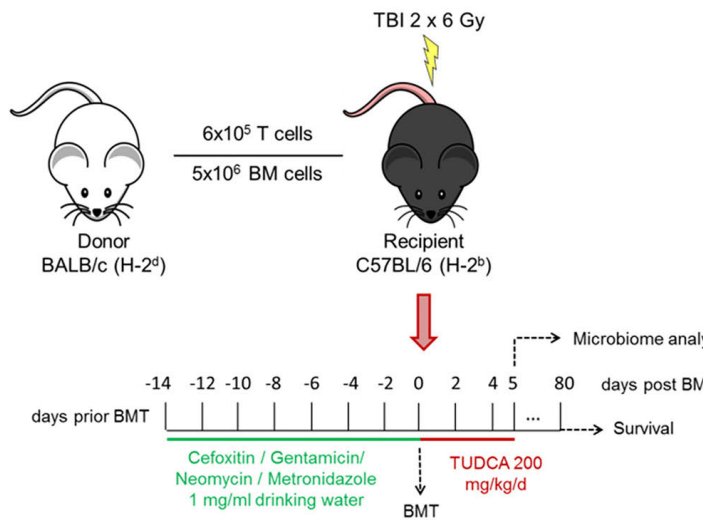

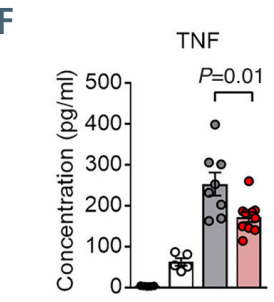

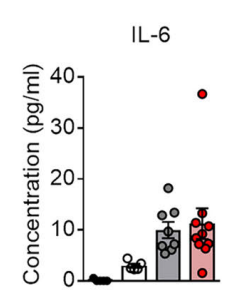

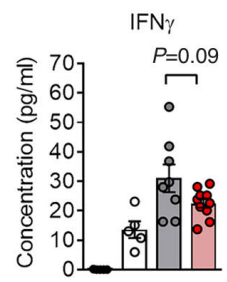

- Untreated $(n=5)$

- $B M(n=5)$

- $B M+T c+$ vehicle $(n=8)$

- $B M+T c+T U D C A(n=10)$
I

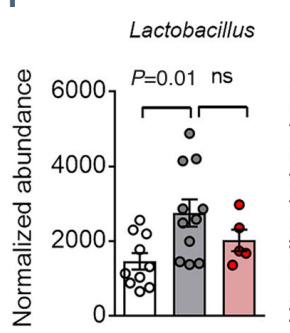

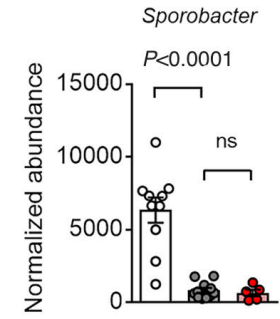

- Untreated $(n=10)$

- $B M+T c+$ vehicle $(n=11)$

- $B M+T c+T U D C A(n=5)$

K

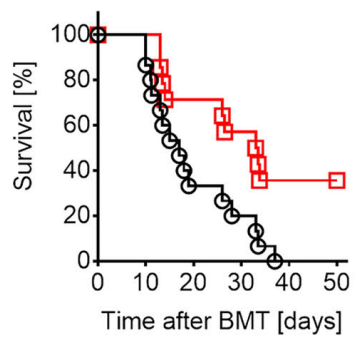

$\left.\begin{array}{l}\ominus B M+T c+\text { antibiotics+vehicle }(n=15) \\ \text { BM+Tc+antibiotics+TUDCA }(n=14)\end{array}\right] P=0.008$

Figure 3. Legend on following page. 
Figure 3. Bile acid treatment improves acute graft-versus-host disease outcome in mice. (A) Transplantation model with BALB/c $(\mathrm{H}-2 \mathrm{kd})$ as donor and $\mathrm{C} 57 \mathrm{BL} / 6$ ( $\mathrm{H}-$ $2 \mathrm{~kb}$ ) as recipient. Recipient animals were treated with $200 \mathrm{mg} / \mathrm{kg}$ body weight tauroursodeoxycholic acid (TUDCA) or an equal volume of vehicle from day 0 until day 10 after bone marrow transplantation (BMT) by a daily intraperitoneal injection. (B) Survival of C57BL/6 mice transplanted as shown in (A). Numbers (N) represent individual mice, the $P$-value was calculated using the two-sided Mantel-Cox test. (C) Transplantation model with C57BL/6 (H-2kb) as donor and BALB/c (H-2kd) as recipient. Recipient animals were treated with $200 \mathrm{mg} / \mathrm{kg}$ body weight TUDCA or an equal volume of vehicle from day 0 until day 10 after BMT by a daily intraperitoneal injection. (D) Survival of BALB/c mice transplanted as described in (C). Data were pooled from three independent experiments, numbers (N) represent individual mice. The $P$-value was calculated using the two-sided Mantel-Cox test. (E) Graft-versus-host disease (GvHD) histopathology scores of liver, small intestine and colon assessed on day 7 after BMT (C57BL/6 in BALB/C model). Data were pooled from two independent experiments, numbers (N) represent individual mice. $P$-values were calculated using the ordinary one-way ANOVA test with correction for multiple comparisons. (F) Serum cytokine concentrations in untreated mice and transplanted mice determined on day 14 after BMT (C57BL/6 in BALB/c model). Numbers $(\mathrm{N})$ represent individual mice. $P$-values were calculated using the ordinary one-way ANOVA test with correction for multiple comparisons. ( $G$ and I) Fecal samples were collected for microbial analysis on day 5 after BMT. Numbers (N) represent individual mice. Data were pooled from two independent experiments. $P$-values were calculated using the ordinary one-way ANOVA test with correction for multiple comparisons; ns: not significant. (G) The Shannon index as a surrogate parameter for microbial diversity. (H) The reversed Simpson index as a surrogate parameter for microbial diversity. (I) Relative abundance of specified bacterial genera. (J) C57BL/6 mice were treated with an antibiotic cocktail comprising $1 \mathrm{mg} / \mathrm{mL}$ cefoxitin metronidazole, neomycin and gentamycin for 2 weeks before they underwent BMT as described in (A). (K) Survival of C57BL/6 mice transplanted and treated as described in $(\mathrm{J})$. Numbers $(\mathrm{N})$ represent individual mice. Data were pooled from two independent experiments. Statistical analysis was performed using the two-sided Mantel-Cox test.

to E). Downregulated genes included transporter associated with antigen processing 1 and 2 (Tap1 and Tap2) (Figure 4B to $\mathrm{E})$, which are involved in the translocation of degraded cytosolic peptides across the endoplasmatic reticulum membrane for antigen-major histocompatibility complex (MHC) class I molecule assembly, as well as TAP binding protein (Tapbp) and Tapasin-related protein (Tapbpt) which mediate the association between TAP proteins and newly assembled MHC class I complexes. Furthermore, lower transcription of class II MHC complex transactivator (Ciita) was observed suggesting decreased MHC class II-related antigen presentation in the intestines of TUDCA-treated animals (Figure $4 \mathrm{~B}$ and $\mathrm{C}$ ). Confirming the hypothesis that the antigen presentation machinery in the intestine is reduced, we found decreased levels of MHC class I and MHC class II expression on non-hematopoietic cells in the intestine of TUDCA-treated animals (Figure 4F). In line with data obtained from the organoid culture system, multiple genes associated with the GO term 'Response to IFN $\gamma$ ' were significantly downregulated (Figure 4G). Interestingly, the numbers of CD11 $\mathrm{c}^{+} \mathrm{MHC}$ class $\mathrm{II}^{+}$professional antigenpresenting cells (APC) in ileum and colon were identical between both groups (Online Supplementary Figure S4A). There was a slight reduction in TNF expression, whereas costimulatory molecules and other cytokines remained unchanged (Online Supplementary Figure S4B and C). The migration capacity of bone marrow-derived dendritic cells (BM-DC) was not impaired by TUDCA treatment either (Online Supplementary Figure S4D). These data support the hypothesis that antigen presentation by non-hematopoietic cells is reduced by TUDCA treatment. In line with this model, treatment with TNF elevated the expression of Tap1 and Tap2 in MODE-K cells which could be reversed by addition of TUDCA (Figure $4 \mathrm{H}$ and I). Notably, the effect on Tap $1 / 2$ expression was most evident for TUDCA but occurred upon treatment with CDCA and UDCA as well (Figure 4H and I).

\section{Bile acid administration changes the transcriptional signature of $T$ cells in the intestine but preserves their systemic expansion}

Antigen presentation is important for the recognition of malignant cells by alloreactive $T$ cells. Since TUDCA reduces antigen presentation in the intestine, we asked whether its application has an immediate impact on T-cell activation. T-cell numbers in the lamina propria of the small intestine and production of IFN $\gamma$, interleukin-6 (IL-6) and TNF were not altered upon TUDCA administration (Figure 5A; Online Supplementary Figure S5A and B). However, we discovered a transcriptional signature suggesting downregulation of immune cell activation. We observed that the transcriptional levels of many genes associated with the GO term 'T-cell activation' were significantly reduced in GvHD developing mice, treated with TUDCA (Figure 5C). Among these genes were the CD3 subunits $\mathrm{CdBe}$ and $\mathrm{Cd} 3 \mathrm{~g}$, the transcription factor interferon regulatory factor 1 (Irf1), as well as many genes encoding for proteins that play an important role for signaling pathways downstream of the T-cell receptor (Figure 5D). Among these were lymphocyte-specific protein tyrosine kinase $(L c k)$ and linker for activation of $\mathrm{T}$ cells $(\mathrm{Lat})$. Lck is a tyrosine kinase that phosphorylates the tails of the $\mathrm{CD} 3$ chains of the T-cell receptor complex upon antigen recognition via $\mathrm{MHC}$. This allows ZAP70 binding and activation. Lat is phosphorylated by ZAP70/Syk kinases upon T-cell receptor activation and recruits adapter proteins which are important for further signaling. This altered transcriptional signature suggests that multiple events in the downstream signaling of the CD3/T-cell receptor complex are reduced upon treatment with TUDCA.

We then investigated whether TUDCA administration could decrease the general alloreactive T-cell expansion. Bioluminescence-based trafficking analysis revealed that $\mathrm{T}$ cell expansion was similar in vehicle- and TUDCA-treated animals (Figure 5E to G). Flow cytometry confirmed that there were no differences in T-cell numbers in the spleen between both groups (Online Supplementary Figure S5C to $F)$. T-cell differentiation, migration and TNF production after in vitro stimulation and TUDCA treatment were comparable (Online Supplementary Figure S5G to I). Collectively, our results support the hypothesis that bile acid application is also associated with a transcriptional signature of reduced local T-cell activation without having a negative impact on systemic T-cell expansion.

\section{Tauroursodeoxycholic acid decreases apoptosis in the intestine}

We observed that bile acids prevent intestinal cell death in vitro. Further analysis of the microarray data obtained from the in vivo allo-HCT model revealed a significant downregulation of apoptosis-related genes in mice treated with TUDCA (Figure 6A). Confirming this, we performed an immunofluorescent TdT-mediated dUTP-biotin nick end labeling (TUNEL) staining which marks apoptotic nuclei. While aGvHD induction significantly increased intestinal apoptosis, TUDCA administration reduced it to almost the baseline level in both the small intestine and the colon (Figure $6 \mathrm{~B}$ and $\mathrm{C}$ ). The application of TUDCA increased the 
expression of the ISC marker genes Lgr5 and Pleckstrin homology-like domain family A member 1 (Phlda1) and of the goblet cell marker Muc2 (Figure 6D). In contrast, no changes in the expression of Defal and Defa 4 or Chga could be identified (Online Supplementary Figure S6A and B). In order to confirm the protection of ISC upon administration of TUDCA, we utilized B6.129P2-Lgr ${ }^{\text {tm1(cre//RT2)Cle/J mice }}$ which express green fluorescent protein (GFP) under control of the Lgr5 gene promoter. On day 14 after transplantation, we detected increased numbers of $\mathrm{GFP}^{+}$cells in the small intestine of TUDCA-treated animals compared to vehicle-treated controls (Figure $6 \mathrm{E}$ and F). Previous studies
A

DOWN in TUDCA
VS. vehicle

B

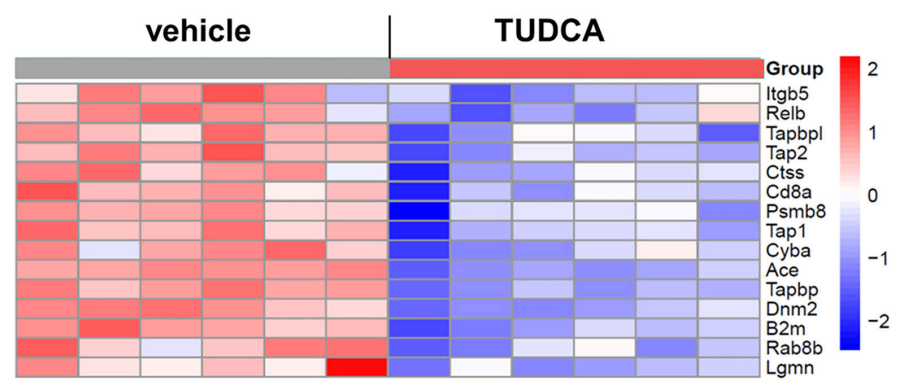

C
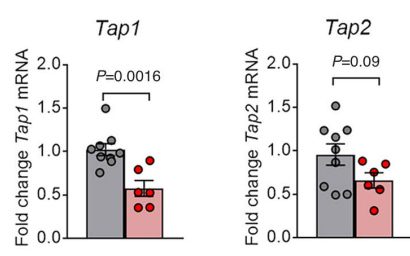

D

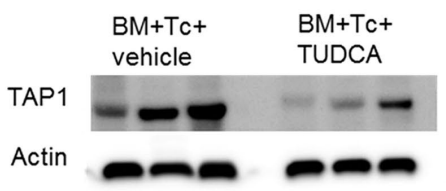

G
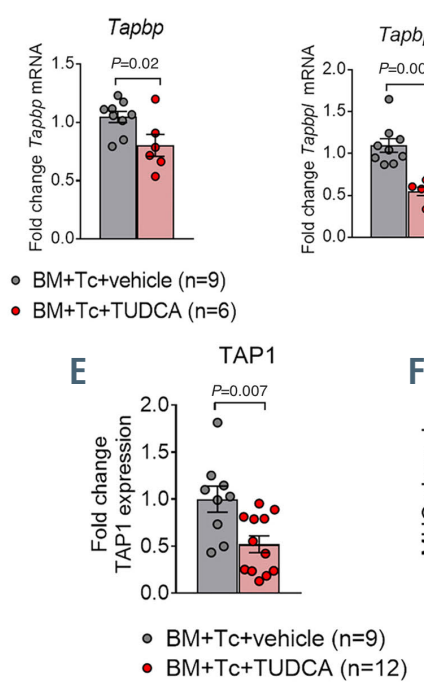
Gene Ontology Response to Interferon gamma

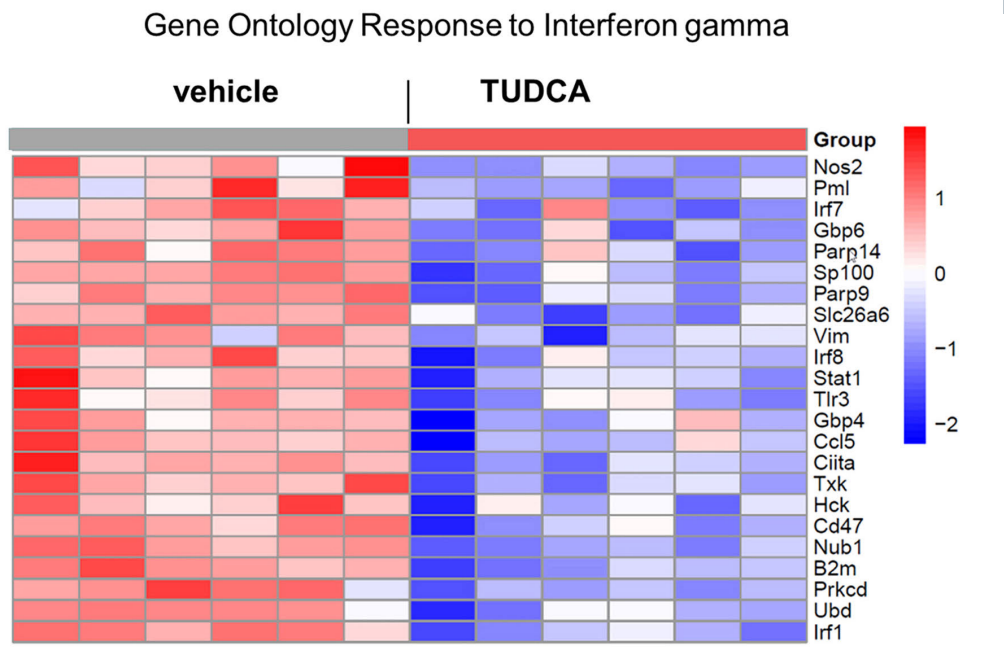

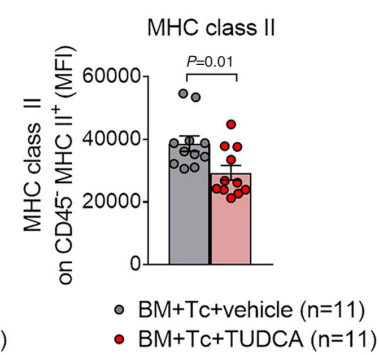

H
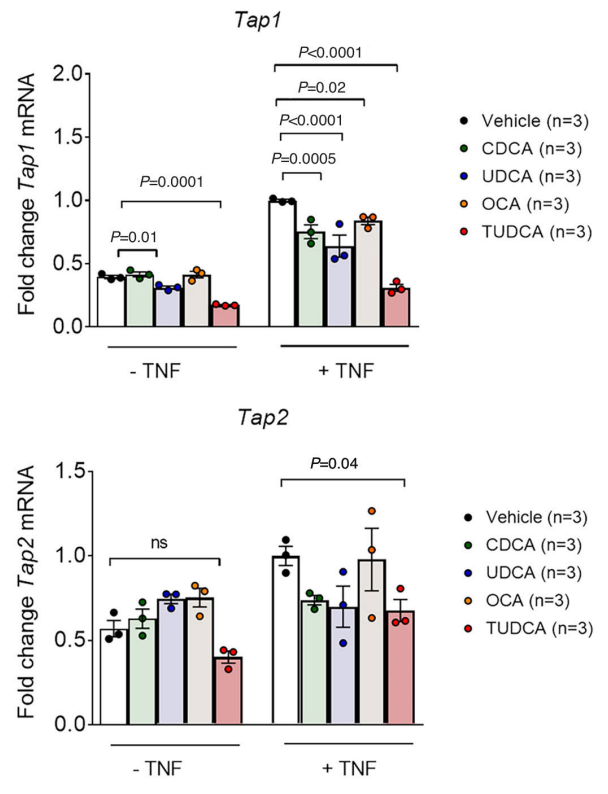
Figure 4. Tauroursodeoxycholic acid reduces intestinal antigen presentation. (A to G) Small intestinal samples were isolated from recipient mice treated with vehicle or tauroursodeoxycholic acid (TUDCA), on day 14 after bone marrow transplantation (BMT) (C57BL/6 to BALB/c model). (A) Identification of significantly downregulated Gene Ontology terms in animals treated with TUDCA. The dotted line corresponds to $P=0.05$ (log $-0.05=1.29$ ). (B) Heat map based on microarray analysis showing the differentially regulated genes ( $q$-value $<0.05$ ) that belong to the term 'antigen processing and presentation' from the Gene Ontology database. Data were pooled from two independent experiments, $n=6$ mice per group, $P=0.004$. The color code represents the Z-score $\log _{2}$ intensity. (C) Quantitative real-time polymerase chain reaction (PCR) analysis of the mRNA expression of selected genes with Actb as a reference gene. Data were pooled from two independent experiments, numbers $(\mathrm{N})$ represent individual mice. $P$-values were calculated using the unpaired two-tailed Student's $t$-test. (D) Expression of TAP1 protein quantified by western blot. Representative western blot from $n=3$ mice per group. (E) Quantification of TAP1 protein expression. Data were pooled from two independent experiments, numbers (N) represent individual mice. $P$-values were calculated using the unpaired two-tailed Student's $t$-test. (F) Flow cytometric quantification of major histocompatibility complex (MHC) class I expression on $\mathrm{CD}_{2} 26^{+}\left(\mathrm{EpCAM}^{+}\right.$) cells (left panel) and $\mathrm{MHC}$ class II expression on $\mathrm{CD}^{-} 5^{-} \mathrm{MHC}$ class II ${ }^{+}$cells (right panel). Data were pooled from two independent experiments, numbers $(\mathrm{N})$ represent individual mice. $P$-values were calculated using the unpaired two-tailed Student's $t$-test. (G) Heat map based on microarray analysis showing the differentially regulated genes (q-value<0.05) that belong to the term 'Response to interferon $\gamma$ ' from the Gene Ontology database. Data were pooled from two independent experiments, $n=6$ mice per group, $P=6.49 \times 10^{-9}$. The color code represents the $Z$-score log ${ }_{2}$ intensity. $(\mathrm{H}$ and $\mathrm{I}$ ) Quantitative real-time PCR analysis of the MRNA expression of Tap1 (panel H) and Tap2 (panel I) with Actb as a reference gene in MODE-K cells treated with TNF \pm chenodeoxycholic acid (CDCA), ursodeoxycholic acid (UDCA), 6-ethylchenodeoxycholic acid (obeticholic acid, OCA) and TUDCA for 48 hours. Representative data from one of two independent experiments with $n=3$ replicates/group are presented. $P$-values were calculated using the ordinary one-way ANOVA test with correction for multiple comparisons; ns: not significant.

had shown a role for TUDCA and its chaperone activity for reducing ER stress, a cellular stress response to critical conditions that can potentially lead to apoptosis. We found no changes of ER stress marker expression in the intestine of mice developing aGvHD when treated with TUDCA (Online Supplementary Figure S6C and D). Altogether, these data demonstrate that TUDCA protects the intestinal tract from apoptosis and particularly preserves ISC and goblet cells from aGvHD-related damage.

\section{Tauroursodeoxycholic acid treatment does not abrogate the graft-versus-Ieukemia activity or hematopoietic regeneration}

We next assessed the impact of TUDCA directly on the graft-versus-leukemia (GvL) effect. Reduced antigen presentation and human leukocyte antigen (HLA) loss contribute to immune escape of acute myeloid leukemia (AML) cells after allo-HCT. ${ }^{25}$ In order to test whether bile acid treatment alters the MHC/TAP antigen presentation in leukemic cells, we treated four human and murine leukemia cell lines with TUDCA. HLA-A, B, C and MHC class I expression were not altered (Figure 7A, B, D and E; Online Supplementary Figure S7). Also Tap1 and Tap2 gene expression remained unchanged, suggesting that bile acid application does not impair the GvL activity by reducing the expression of $\mathrm{MHC} / \mathrm{TAP}$ molecules on malignant cells (Figure 7C and F; Online Supplementary Figure S7).

We then activated $\mathrm{CD}^{+} \mathrm{T}$ cells by co-incubation with allogeneic dendritic cells and treated them with TUDCA prior to incubation with the A20 lymphoma cell line. T cells were capable of inducing cell death in the leukemic cells which remained stable in the case of TUDCA addition (Figure 7G). We tested in vivo T-cell priming by re-isolating $\mathrm{T}$ cells from the spleens of vehicle- and TUDCA-treated animals 14 days after BMT (Figure $7 \mathrm{H}$ ). These in vivo activated $T$ cells had a comparable killing capacity when incubated with A20 cells (Figure 7I). Finally, we studied the GvL effect in vivo by injecting $\mathrm{Ba} / \mathrm{F3}$ cells containing the FLT3ITD translocation (Ba/F3-ITD) (Figure 7J). Additional transfer of alloreactive $\mathrm{T}$ cells reduced the expansion of the malignant cells in the bone marrow and the spleen. This effect persisted in the mice upon TUDCA treatment (Figure $7 \mathrm{~K})$. These data indicate that TUDCA affects specifically the intestine and does not impede cytotoxic lymphocyte activity against malignant cells.

Since immune reconstitution is critical for a favorable outcome after allo-HCT, we investigated whether TUDCA might have a negative impact on the peripheral blood cell reconstitution. Mice developing aGvHD treated with
TUDCA and vehicle displayed the expected decrease in hemoglobin, hematocrit, platelets and white blood cells (WBC) in comparison to untreated mice (Online Supplementary Figure S8A). TUDCA did not enhance cytopenia as treated animals exhibited the same blood counts as vehicle controls. WBC subpopulation analysis by flow cytometry revealed similar proportions of $\mathrm{T}$ cells, B cells, granulocytes and monocytes in the peripheral blood (Online Supplementary Figure S8B). Analysis of mice with aGvHD at a later time point was not possible due to GvHD-induced mortality in the vehicle group. In order to segregate aGvHD from immune reconstitution, we analyzed animals transplanted only with BM without a GvHDinducing transfer of $\mathrm{T}$ cells. TUDCA treatment did not compromise hematopoietic regeneration (Online Supplementary Figure S8C and D). These results underline the observation that TUDCA treatment does not prolong the time period necessary for immune reconstitution and suggest that this compound would not impair immune responses to pathogens or the GvL response in allo-HCT patients.

\section{Discussion}

Frequency of allo-HCT is rising worldwide since improved conditioning protocols and supportive care allow older patients to undergo this potentially curative leukemia treatment. However, efficient GvHD prophylaxis remains a challenge in the clinical routine with a significant impact on the long-term outcome of allo-HCT.

Here, we observed a depletion of the bile acid pool after allo-HCT. We hypothesized that loss of bile acids might be one of the factors contributing to loss of homeostasis in the intestinal tissue during GvHD (Figure 8). In agreement with recently published data, ${ }^{6,7}$ we observed that exposure to pro-inflammatory cytokines caused intestinal tissue damage in an organoid culture model. Treatment with bile acids improved the viability of intestinal organoids and cell lines when cell death was induced by application of TNF or IFN $\gamma$. The role of bile acids in the regulation of cell death has been controversially discussed. ${ }^{26}$ Depending on their concentration, they can have cytotoxic properties and are able to induce apoptosis either by direct activation of death receptors, for example FAS, or by inducing oxidative damage and mitochondrial dysfunction. ${ }^{27-29}$ Other studies indicate that there is a fine balance in bile acid composition in order for their toxic and protective properties to antagonize one another. $^{30,31}$ The secondary hydrophilic bile acid UDCA has 
A

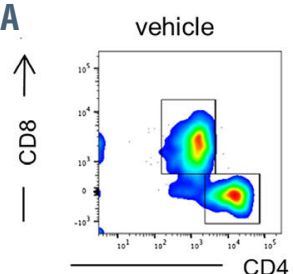

TUDCA

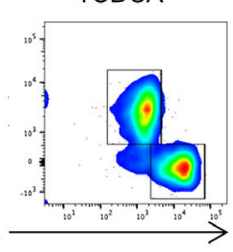

B

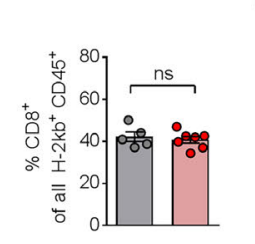

\section{Ileum}

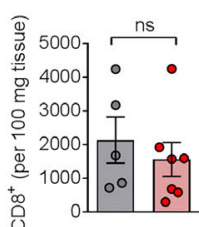

- $B M+T c+v e h i c l e ~(n=5)$

- BM+Tc+TUDCA $(n=6-7)$

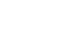

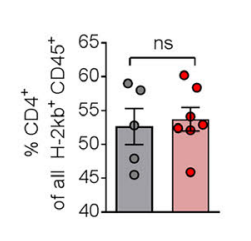

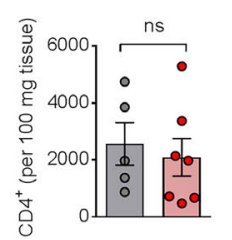

- $B M+T c+$ vehicle $(n=5)$

- $B M+T c+T U D C A(n=6-7)$

C

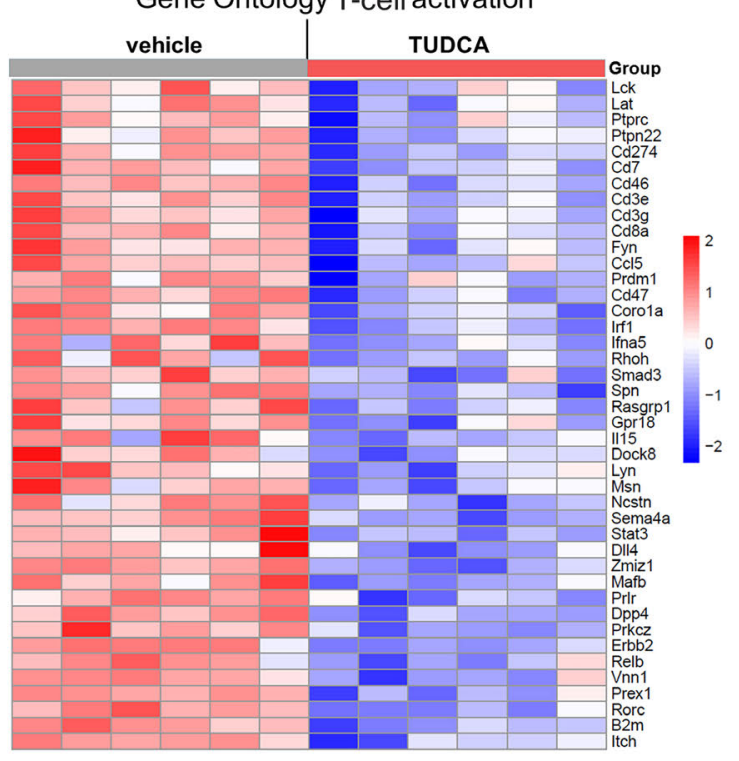

D
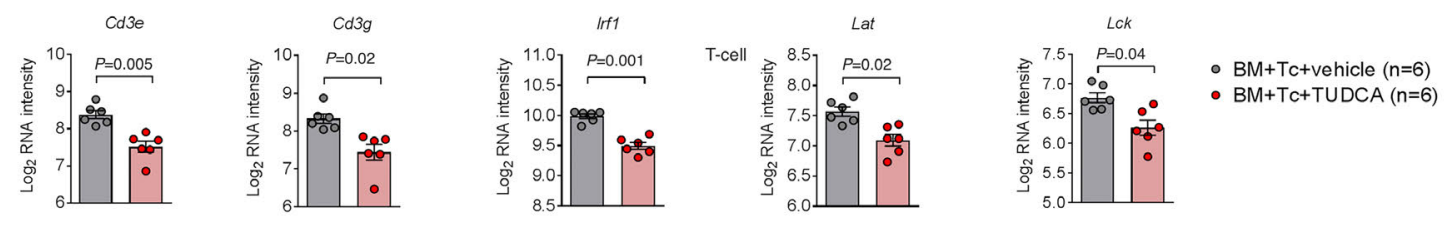

E

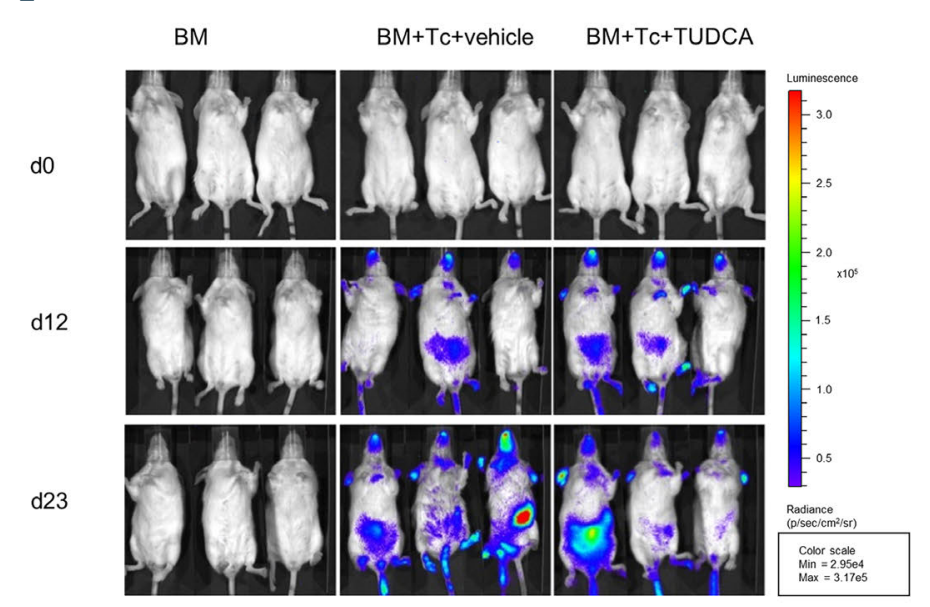

F

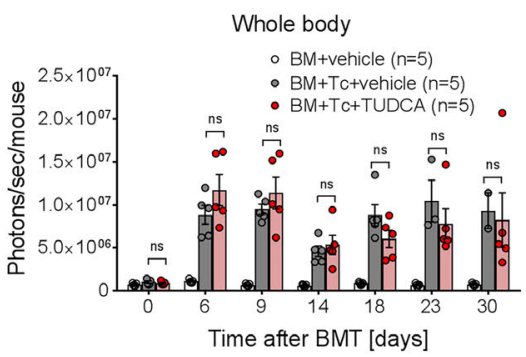

G

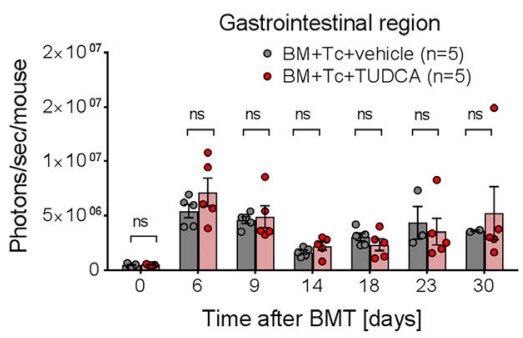

Figure 5. Tauroursodeoxycholic acid administration changes the transcriptional signature of T cells in the intestine but preserves their systemic expansion. (A and B) Flow cytometric analysis of $\mathrm{T}$ cells isolated from the small intestinal lamina propria of recipient animals on day 14 after bone marrow transplantation (BMT) (C57BL/6 in BALB/c model). (A) Representative flow cytometry dot plots. (B) Relative (left panel) and absolute (right panel) quantification of CD8 ${ }^{+}$and CD4 ${ }^{+}$T cells. Representative data from one of two biologically independent experiments, numbers $(\mathrm{N})$ represent individual mice. $P$-values were calculated using the two-tailed unpaired Student's $t$-test; ns: not significant. (C) Heat map based on microarray analysis (performed as in Figure 4) showing the differentially regulated genes (qvalue $<0.05$ ) that belong to the term 'T-cell activation' from the Gene Ontology database. Data were pooled from two independent experiments, $\mathrm{n}=6 \mathrm{mice}$ per group, $P=4.6 \times 10^{-11}$. The color code represents the Z-score $\log _{2}$ intensity. (D) $\log _{2}$ RNA intensity values for selected genes belonging to the GO term “T-cell activation". Data were pooled from two independent experiments, numbers $(N)$ indicate individual mice. An adjusted $P$-value calculated as described in the Methods is presented. (E) BALB/c mice underwent transplantation as described in Figure $3 \mathrm{C}$ using luciferase-transgenic T cells. Representative bioluminescence images for T-cell trafficking on different time points after transplantation. ( $F$ and $G$ ) Quantification of the bioluminescence measurement performed as described in (D). Signal was quantified either from the whole body $(F)$ or from the gastrointestinal region only $(G)$. One of three independent experiments with $n=5$ mice per group is shown. $P$-values were calculated using the ordinary one-way ANOVA test with correction for multiple comparisons. 
A Consensus Apoptosis Gene Set
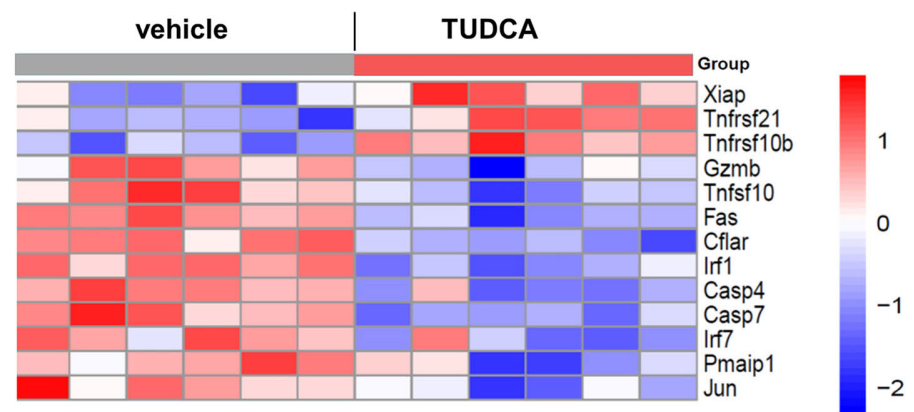

B

Untreated

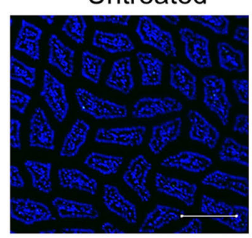

$\mathrm{BM}+\mathrm{Tc}+\mathrm{vehicle}$

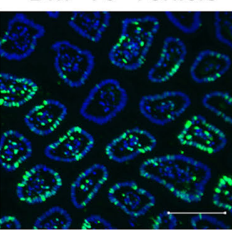

Small intestine; Blue: DAPI; Green: apoptotic nuclei
C

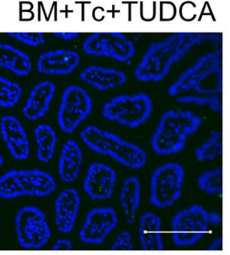

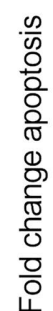

Small intestine

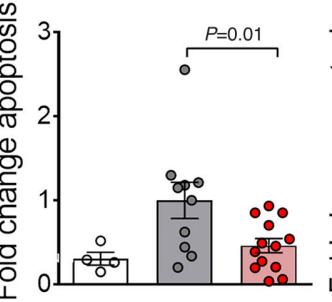

Colon

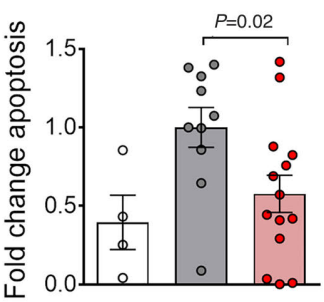

- Untreated $(n=4)$

- $B M+T c+$ vehicle $(n=10)$

- BM+Tc+TUDCA $(n=13)$

D

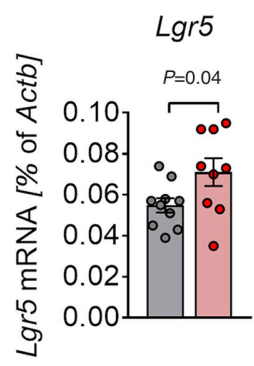

Phlda1

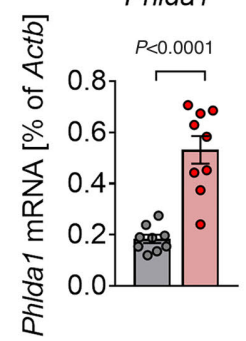

Muc2

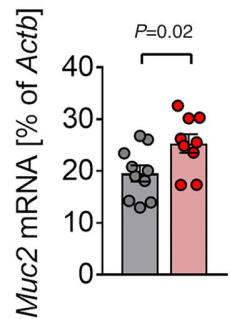

- $B M+T c+$ vehicle $(n=10)$

- BM+Tc+TUDCA $(n=9)$

E
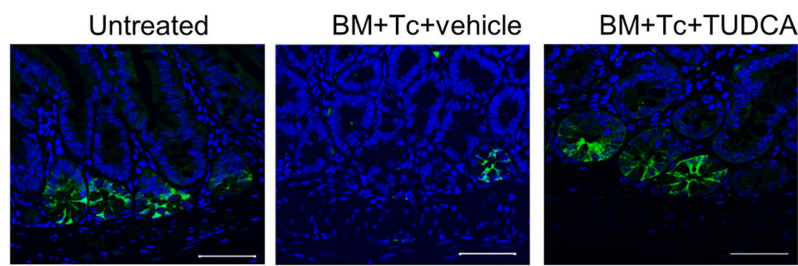

Small intestine, Lgr5-GFP reporter mice

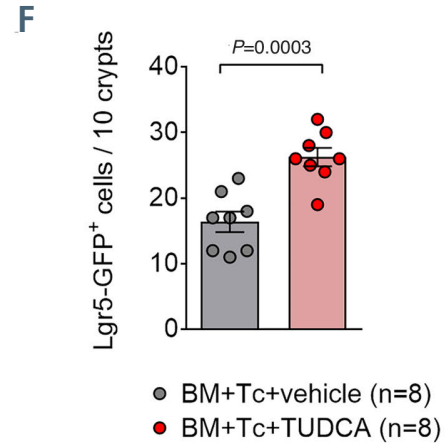

F

- $B M+T c+T U D C A(n=8)$

\begin{abstract}
Figure 6. Tauroursodeoxycholic acid decreases intestinal apoptosis. (A to D) Small intestinal samples were isolated from recipient mice which were treated with vehicle or tauroursodeoxycholic acid (TUDCA), on day 14 after bone marrow transplantation (BMT) (C57BL/6 to BALB/c model). (A) Heat map based on microarray analysis showing the differentially regulated genes (q-value<0.05) from the gene set 'apoptosis' from the CONSENSUS database (small intestine, day 14 after BMT). Data were pooled from two independent experiments, $n=6$ mice per group, $P=0.001$. The color code represents the Z-score log intensity. (B) Representative immunofluorescence images of TUNEL-stained paraffin sections from the small intestine (day 14 after BMT, blue: DAPI, green: apoptotic TUNEL staining). Scale bars 200 um. (C) Quantification of apoptotic nuclei in the small intestine and the colon was obtained using the Olympus ScanR analysis software (day 14 after BMT). Data were pooled from two independent experiments, numbers $(\mathrm{N})$ indicate individual mice. P-values were calculated using the ordinary one-way ANOVA test with correction for multiple comparisons. (D) Quantitative real-time polymerase chain reaction (PCR) analysis of the mRNA expression of the intestinal stem cell markers Lgr5 and Phlda1 and the goblet cell marker Muc2 with Actb as a reference gene (small intestine, day 14 after BMT). Data were pooled from two independent experiments,

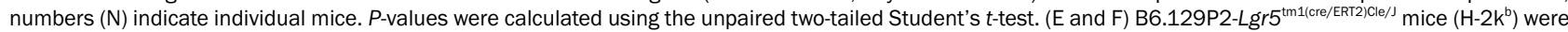
transplanted and treated with TUDCA as described in Figure 3A. On day 14 after BMT, the small intestine was analyzed by immunofluorescence for the number of green fluorescent protein positive (GFP ${ }^{+}$intestinal stem cells. (E) Representative images obtained using confocal microscopy (blue: DAPI, green: GFP). Scale bars 50 um. (F) Quantification of data pooled from two independent experiments, $N$ numbers represent individual mice. The $P$-value was calculated using the unpaired twotailed Student's $t$-test.
\end{abstract}


A

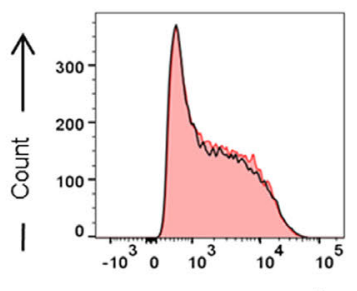

D

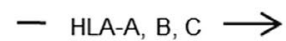

RMB-1

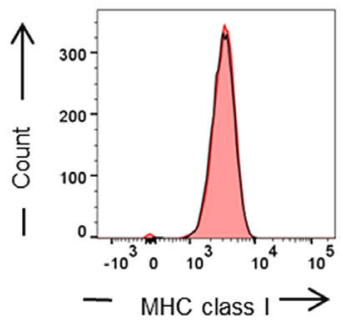

G

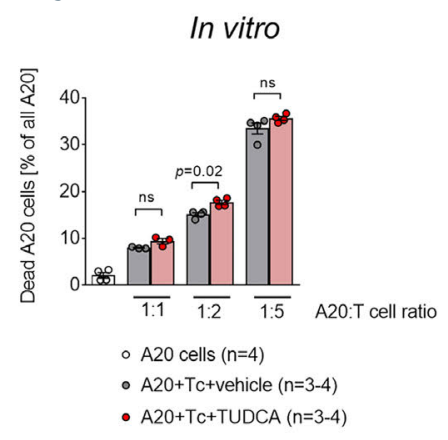

J

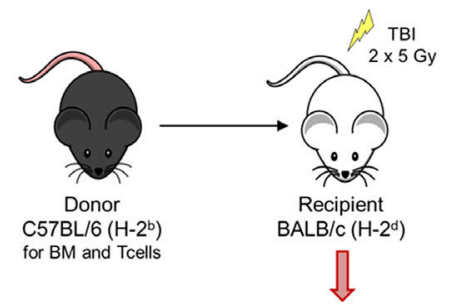

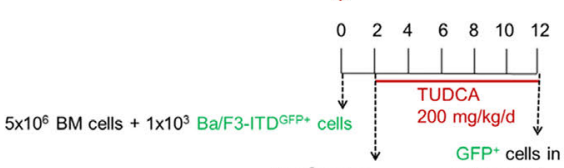

$3 \times 10^{5}$ Tcells

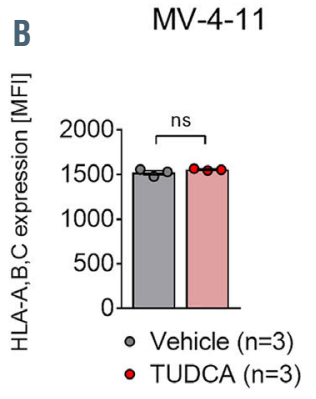

E
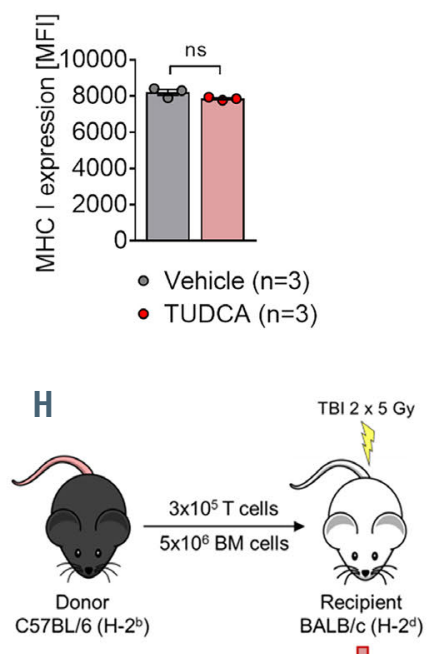

1
$\mathrm{H}$

H

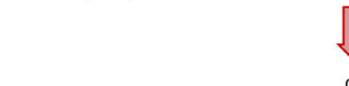

C

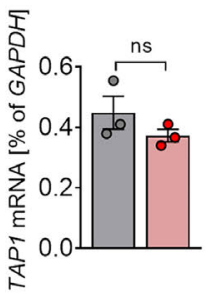

$\mathbf{F}$

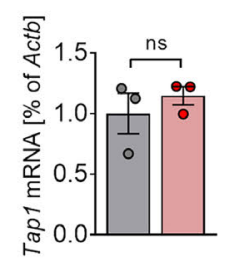

MV-4-11

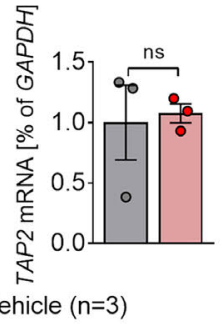

- $\operatorname{TUDCA}(n=3)$

RMB-1

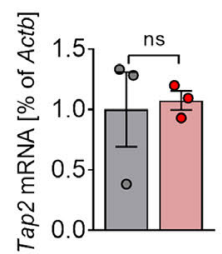

- Vehicle $(n=3)$

- TUDCA $(n=3)$

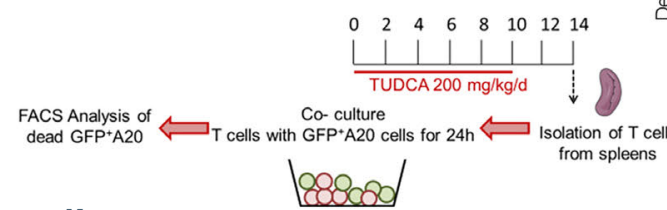

I

\section{Ex vivo}

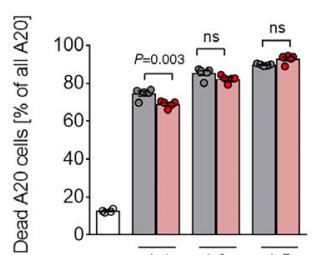

$\overline{1: 1} \quad \overline{1: 2} \quad \overline{1.5} \quad$ A20:T cell ratio

- A20 cells ( $\mathrm{n}=4$ )

- A20+Tc+vehicle $(n=5)$

- $A 20+T c+T U D C A(n=5)$

\section{K}
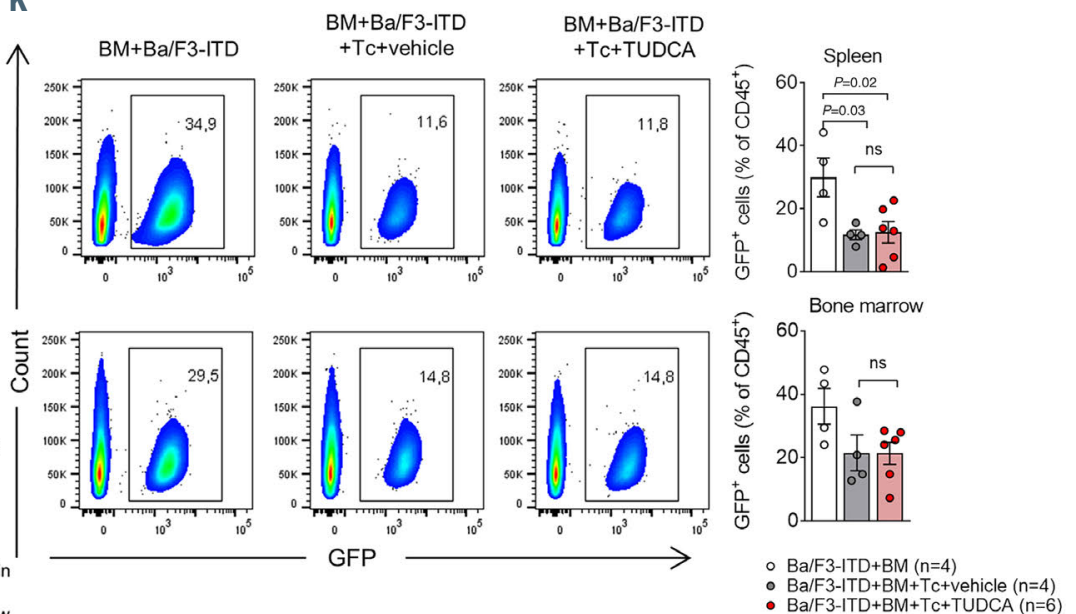

Figure 7. The graft-versus-leukemia/lyphoma effect is preserved despite tauroursodeoxycholic acid administration. (A to F) MV-4-11 and RMB-1 cells were cultured for 72 hours (h) with or without addition of tauroursodeoxycholic acid (TUDCA). One representative result from three independent experiments performed in technical triplicates is shown for panel (A) and (D). In panels $(B),(C),(E)$ and $(F)$ data were pooled from three independent experiments performed in technical duplicates or triplicates. P-values were calculated using the two-tailed unpaired Student's $t$-test; ns: not significant. (A) Representative histograms from flow cytometric analysis of human leukocyte antigen (HLA) A, B, C expression on MV-4-11 cells. (B) Quantification of HLA A, B and C expression on MV-4-11 cells. (C) Quantitative polymerase chain reaction (PCR) analysis of the expression of the antigen presentation-related genes TAP1 and TAP2 in MV-4-11 cells with glyceraldehyde 3-phosphate dehydrogenase (GAPDH) as reference gene. (D) Representative histograms from a flow cytometric analysis of major histocompatibility complex (MHC) class I expression on RMB-1 cells. (E) Quantification of MHC class I expression on RMB-1 cells. (F) Quantitative PCR analysis of the expression of the antigen presentation-related genes Tap1 and Tap2 in RMB-1 cells with Actb as reference gene. (G) C57BL/ 6 CD8 ${ }^{+} \mathrm{T}$ cells were activated with allogeneic (BALB/c) dendritic cells for $72 \mathrm{~h}$ and treated with $500 \mu \mathrm{M}$ TUDCA or vehicle prior to incubation with A20 lymphoma cells. The percentage of dead A20 cells after $24 \mathrm{~h}$ of incubation was analyzed by flow cytometry. 
Representative data from one of two biologically independent experiments performed with three to four technical replicates respectively. (H) Experimental model for assessing the graft-versus-ligand (GvL) response of allogeneic T cells ex vivo. BALB/c mice underwent bone marrow transplantation (BMT) as described in Figure $3 \mathrm{C}$ and T cells from spleens were isolated for subsequent co-culture with A20 cells on day 14 after BMT. (I) Flow cytometric quantification of dead A20 lymphoma cells co-cultured with $\mathrm{CD} 4^{+}$and $\mathrm{CD} 8^{+} \mathrm{T}$ cells re-isolated from the spleens of recipient mice on day 14 after BMT as described in (H). Representative data from one of two biologically independent experiments performed with four to five technical replicates respectively. $P$-values were calculated using the ordinary one-way ANOVA test with correction for multiple comparisons, ns: not significant. $(J)$ Experimental model for assessing the GvL response in vivo. BALB/c mice underwent BMT with additional injection of green fluorescent protein positive $\left(\mathrm{GFP}^{+}\right) \mathrm{Ba} / \mathrm{F} 3-\mathrm{ITD}$ leukemia cells. Allogeneic T cells were transferred two days later and animals were treated with $200 \mathrm{mg} / \mathrm{kg}$ body weight TUDCA or vehicle for another 10 days. (K) Flow cytometric analysis of spleen and bone marrow for the percentage of GFP ${ }^{+}$cells on day 12 after tumor injection. $\mathrm{N}$ numbers represent individual mice. Left panel: representative flow cytometry plots. Right panel: quantification, numbers (N) represent individual mice. P-values were calculated using the ordinary one-way ANOVA test with correction for multiple comparisons, ns: not significant.

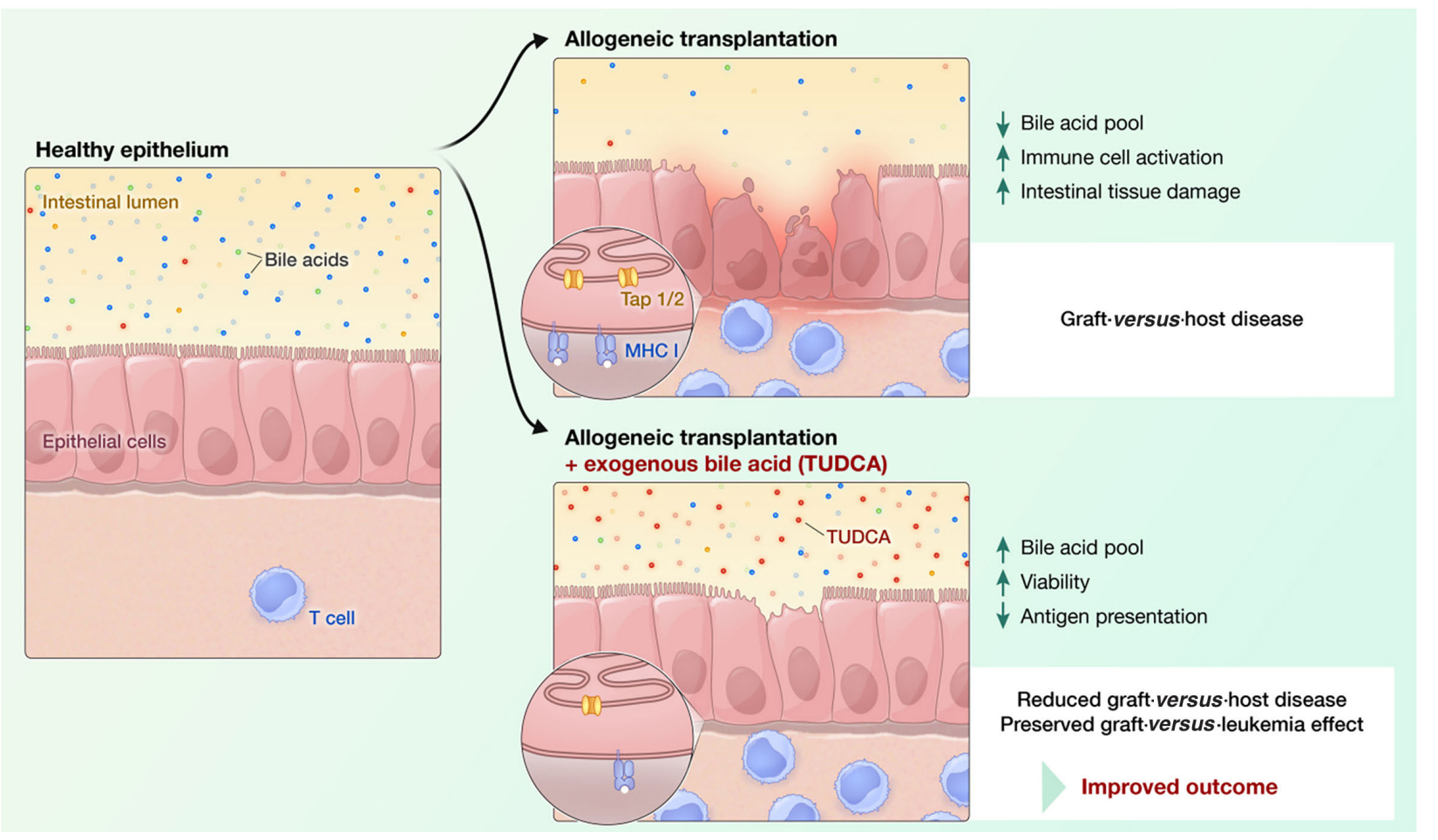

Figure 8. Tauroursodeoxycholic acid leads to a reduction of acute graft-versus-host disease. A model, in which this positive effect is achieved by two distinct mechanisms: enhancing the viability during exposure to pro-inflammatory cytokines and reduction of antigen presentation in the intestine with a consequent decrease in apoptosis.

cytoprotective functions. UDCA is approved for the treatment of patients with cholestatic liver diseases based on inhibition of hepatocyte apoptosis and protection from toxic hydrophobic bile acids. ${ }^{26,32}$ These effects are linked to a stabilization of the mitochondria, reduced BAX translocation and decreased cytochrome $\mathrm{C}$ release and subsequent apoptosis. ${ }^{32,33}$ Furthermore, the administration of TUDCA led to the inhibition of cellular damage introduced by the bile acid glycochenodeoxycholic acid (GCDCA) by preventing GCDCA-induced caspase- 9 activation and subsequent mitochondrial damage. Therefore, such bile acids can enable survival via protection against more hydrophobic and potentially more toxic bile acid variants. ${ }^{34,35}$ Also a protection of hepatocytes from carcinogen-induced apoptosis ${ }^{36}$ and of renal tubular cells against contrast media-induced apoptosis ${ }^{37}$ has been described.

Translating these data into a preclinical BMT model, we observed that application of the bile acid TUDCA, the most potent agent in our in vitro studies, prolonged the survival of mice with aGvHD. Exogenous bile acid application was able to substantially modulate the bile acid pool. TUDCA increased to $35 \%$ of all measured bile acids in the serum and almost $60 \%$ of the bile acids measured in the ileal con- tent. These data suggest that exogenous application is effective in changing the bile acid pool and therefore probably counteracts the depletion of bile acids observed upon GvHD induction. The prophylactic use of UDCA has been previously proposed in a study which demonstrated a reduction in aGvHD incidence as well as non-relapse mortality with a benefit in overall survival. ${ }^{38}$ However, other studies failed to confirm this. ${ }^{39,40}$ Overall, a meta-analysis including four prospective trials and two historical analyses of prophylactic UDCA use in allo-HCT recipients showed a reduction in the levels of hepatic veno-occlusive disease and transplant-related mortality, with no statistically significant difference in the incidence of acute hepatic GvHD or overall survival. ${ }^{41}$ The impact on intestinal aGvHD incidence was not evaluated. Our observations prompted us to search for a mechanism by which bile acids and TUDCA in particular might protect the intestinal epithelium from an alloimmunity-mediated damage. Administration of TUDCA led to reduced expression of antigen presentationrelated genes and to reduced expression of $\mathrm{MHC}$ class I and II on subpopulations of non-hematopoietic cells in the intestine. It has recently been shown that MHC class IIexpressing intestinal epithelial cells are able to present anti- 
gen and activate $\mathrm{T}$ cells in the context of GvHD. ${ }^{42}$ Little is known about the connection of bile acids and their effect on antigen-presentation, especially in non-hematopoietic cells. In a model of Schistosoma mansoni infection, 24-norUDCA but not UDCA itself reduced surface MHC class II expression on macrophages and dendritic cells and the activation as well as proliferation of $\mathrm{T}$ lymphocytes in vitro. ${ }^{43}$ Microbial diversity is a key factor in regulating the homeostasis of the intestine. GvHD has been previously linked to alterations in the microbiome with loss of bacterial diversity. In order to prove that TUDCA regulates aGvHD severity by acting directly on the intestinal epithelium and not by changing the microbiome, ${ }^{44}$ we analyzed fecal samples of TUDCA-treated animals developing aGvHD. Diversity and abundancy analyses showed comparable results between vehicle- and TUDCA-treated mice underlining the hypothesis that TUDCA protects the intestinal epithelium in a cellspecific manner.

Current GvHD prophylaxis and treatment mostly rely on suppression of T-cell activation and cytokine release which on the downside increases the risk of malignancy relapse. While TUDCA treatment resulted in a decreased expression of genes related to T-cell activation in the intestine, it did not impair systemic T-cell proliferation or the activity against tumor cells. This difference might be explained by the fact that bile acid levels are much more abundant in the intestine than in the systemic blood circulation $(0.5 \mu \mathrm{g} / \mathrm{mL}$ in the serum vs. $1,000 \mu \mathrm{g} / 100 \mathrm{mg}$ ileal content). Furthermore, the expression of antigen presentation-related proteins on leukemic cells was not reduced showing that the recognition of malignant cells by the immune system remains intact upon bile acid administration. In line with this concept, we showed preserved GvL activity in in vitro, ex vivo and in vivo models.

One potential explanation for the divergent effects of bile acids on intestinal and leukemic cells is the differential expression of bile acid receptors and their affinities to different species of bile acids. Intestinal epithelial cells are equipped with various bile acid receptors, FXR and TGR5 amongst others. ${ }^{45}$ We show that mRNA expression of both receptors changes upon GvHD induction with an increase of FXR and decrease of TGR5. One could speculate that these alterations in receptor expression make bile acid signaling a potential target for regulating intestinal homeostasis. Both bile acid receptors are able to bind bile acids with a high affinity and subsequently induce different signaling pathways including inflammation and apoptosis signaling. Other receptors of bile acids are more likely to be acknowledged as bile acid "sensors" which rather chemically convert bile acids and can act on bile acid transporters. ${ }^{46} \mathrm{FXR}$ activation in the intestine has several anti-inflammatory properties. OCA, as a strong semi-synthetic FXR agonist, was shown to ameliorate intestinal mucosal inflammation in several mouse colitis models via induction of antimicrobial peptide production and reinforcement of epithelial barrier function in intestinal epithelial cells. ${ }^{47}$ Since OCA and CDCA, the bile acids with the highest affinity to FXR, showed only minor protective effects compared to TUDCA on intestinal epithelium in our experiments, we hypothesize that there must be other underlying mechanisms than the engagement of FXR signaling. One other candidate is the high affinity receptor TGR5 which also induces antiinflammatory responses similar to $\mathrm{FXR}^{48}$ but has the highest affinity to secondary bile acids such as UDCA and its taurine conjugate TUDCA and is expressed in the liver and intestine. 99,50

Amongst our tested bile acids, TUDCA was the one with the strongest anti-apoptotic effects both in vitro as and in vivo. Active re-absorption of bile acids occurs in the terminal ileum and is dependent on their conjugation status. During this process, bile acids come in contact and modulate the mucosal immune system. ${ }^{19,20}$ Our results indicate that for GvHD prevention, bile acids have to be conjugated in order to fulfill their cytoprotective properties.

In summary, we show that allo-HCT induces a depletion of the bile acid pool. Exogenous application of bile acids and in particular TUDCA reduces aGvHD. We propose a model, in which this positive effect is achieved by two distinct mechanisms: enhancing the viability during exposure to pro-inflammatory cytokines and reduction of antigen presentation in the intestine with a consequent decrease in apoptosis (Figure 8). A major advantage of TUDCA is that it is already approved by the Food and Drug Administration and that it possesses a good safety profile. These findings pave the way for a prospective clinical trial using TUDCA to improve the outcome of allo-HCT recipients by preventing aGvHD and preserving the GvL effect.

\section{Disclosures}

$E L P$ is the founder of Rheos Medicines and is a SAB member of Immunomet.

\section{Contributions}

$E H$ and FMU helped to develop the concept, performed experiments, analyzed data and helped to write the manuscript; $G A$ analyzed microarray data; $M P$ and $A B$ performed the microbiome experiments and analysis; $B S$ performed experiments and analyzed data; $L M B$ and EVG performed research; PRE and SFM helped to develop the overall concept and to write the manuscript; DP performed and analyzed microarray experiments; MF helped with microscopy experiments, imaging and data analysis; ASG performed histopathological analysis; $J B$ and ELP performed metabolomics experiments and helped with data analysis; JD and BG helped to design the study and analyze the data; $M B$ analyzed microarray data and helped to write the manuscript; $R Z$ and PA designed the study, planned the experiments and analyzed data; $P A$ also performed experiments and wrote the manuscript.

\section{Acknowledgments}

The authors would like to acknowledge Dr. D. Kaiserlian (INSERM, France) who kindly provided the MODE-K cells. The authors would further like to acknowledge Michal Rössler for designing the graphical abstract.

\section{Funding}

This study was supported by the Else Kröner-Fresenius-Stiftung (EKFS 2015_A147 to PA), the INTERREG V European regional development fund (European Union) program (project 3.2 TRIDIAG to RZ), Wilhelm Sander Stiftung (grant 2008.046.5 to RZ), Deutsche Forschungsgemeinschaft, Germany, SFB1160 (RZ) and SFB850 (MB), ERC Consolidator grant (681012 GvHDCure European Union, to $R Z$ and by the Excellence Strategy of the German Federal and State Governments (CIBSS - EXC 2189). PA is supported by a scholarship from the Berta Ottenstein Program for Physician Scientists, Faculty of Medicine, Medical Center - University of Freiburg, Germany. MB is supported by the German Federal Ministry of Education and Research (BMBF) within the framework of the e:Med research and funding concept 
(CoNfirm, FKZ 01ZX1708F) and within the Medical Informatics Funding Scheme (MIRACUM, FKZ 01ZZ1606A-H). BG receives support through the Deutsche Forschungsgemeinschaft (DFG) SFB1160/2_B5, under Germany's Excellence Strategy (CIBSS - EXC-2189 - Project ID 390939984, and RESIST -
EXC 2155 - Project ID 39087428); through the E-rare program of the EU, managed by the DFG, grant code GR1617/141/iPAD; and through the "Netzwerke Seltener Erkrankungen" of the German Ministry of Education and Research (BMBF), grant code: GAIN 01GM1910A.

\section{References}

1. de Lima M, Porter DL, Battiwalla M, et al. Proceedings from the National Cancer Institute's Second International Workshop on the biology, prevention, and treatment of relapse after hematopoietic stem cell transplantation: part III. Prevention and treatment of relapse after allogeneic transplantation. Biol Blood Marrow Transplant. 2014;20(1):4-13

2.Zeiser R, Blazar BR. Acute Graft-versusHost Disease - Biologic Process, Prevention, and Therapy. N Engl J Med. 2017; 377(22):2167-2179.

3. McDonald GB. How I treat acute graft-versus-host disease of the gastrointestinal tract and the liver. Blood. 2016;127(12):15441550.

4. Takashima S, Kadowaki M, Aoyama K, et al. The Wnt agonist R-spondin1 regulates systemic graft-versus-host disease by protecting intestinal stem cells. J Exp Med. 2011;208(2):285-294.

5. Eriguchi Y, Takashima S, Oka $\mathrm{H}$, et al. Graft-versus-host disease disrupts intestinal microbial ecology by inhibiting Paneth cell production of alpha-defensins. Blood. 2012;120(1):223-231.

6. Eriguchi Y, Nakamura K, Yokoi Y, et al. Essential role of IFN-gamma in T cell-associated intestinal inflammation. JCI Insight. 2018;3(18):e121886

7. Takashima S, Martin ML, Jansen SA, et al. $T$ cell-derived interferon-gamma programs stem cell death in immune-mediated intestinal damage. Sci Immunol. 2019;4 (42): eaay8556.

8. Monte MJ, Marin JJ, Antelo A, VazquezTato J. Bile acids: chemistry, physiology, and pathophysiology. World Gastroenterol. 2009;15(7):804-816.

9. Russell DW. The enzymes, regulation, and genetics of bile acid synthesis. Annu Rev Biochem. 2003;72:137-174

10. Benz C, Angermuller S, Tox U, et al. Effect of tauroursodeoxycholic acid on bile-acidinduced apoptosis and cytolysis in rat hepatocytes. J Hepatol. 1998;28(1):99-106.

11. Sola S, Aranha MM, Steer CJ, Rodrigues CM. Game and players: mitochondrial apoptosis and the therapeutic potential of ursodeoxycholic acid. Curr Issues Mol Biol. 2007:9(2):123-138.

12. Colell A, Coll O, Garcia-Ruiz C, et al. Tauroursodeoxycholic acid protects hepatocytes from ethanol-fed rats against tumor necrosis factor-induced cell death by replenishing mitochondrial glutathione. Hepatology. 2001;34(5):964-971.

13. Castro RE, Sola S, Ramalho RM, Steer CJ, Rodrigues CM. The bile acid tauroursodeoxycholic acid modulates phosphorylation and translocation of bad via phosphatidylinositol 3-kinase in glutamateinduced apoptosis of rat cortical neurons. J Pharmacol Exp Ther. 2004;311(2):845-852.

14. Ishigami F, Naka S, Takeshita K, Kurumi Y, Hanasawa K, Tani T. Bile salt tauroursodeoxycholic acid modulation of Bax translocation to mitochondria protects the liver from warm ischemia-reperfusion injury in the rat. Transplantation. 2001;72 (11):1803-1807

15. Vavassori P, Mencarelli A, Renga B, Distrutti E, Fiorucci $S$. The bile acid receptor FXR is a modulator of intestinal innate immunity. J Immunol. 2009;183(10):6251 6261.

16. Joshi NM, Hassan S, Jasani P, et al. Bile acid malabsorption in patients with graft-versus-host disease of the gastrointestinal tract. Br J Haematol. 2012;157(3):403-407.

17. Michonneau D, Latis E, Curis E, et al. Metabolomics analysis of human acute graft-versus-host disease reveals changes in host and microbiota-derived metabolites. Nat Commun. 2019;10(1):5695.

18. Sato T, Clevers H. Primary mouse small intestinal epithelial cell cultures. Methods Mol Biol. 2013;945:319-328.

19. Ridlon JM, Kang DJ, Hylemon PB. Bile salt biotransformations by human intestinal bacteria. J Lipid Res. 2006;47(2):241-259.

20. Camilleri M. Bile Acid diarrhea: prevalence, pathogenesis, and therapy. Gut Liver 2015;9(3):332-339

21. Swann JR, Want EJ, Geier FM, et al Systemic gut microbial modulation of bile acid metabolism in host tissue compartments. Proc Natl Acad Sci U S A. 2011;108 Suppl 1:4523-4530.

22. Jenq RR, Ubeda C, Taur Y, et al. Regulation of intestinal inflammation by microbiota following allogeneic bone marrow transplantation. J Exp Med. 2012;209(5):903911.

23. Holler E, Butzhammer P, Schmid K, et al. Metagenomic analysis of the stool microbiome in patients receiving allogeneic stem cell transplantation: loss of diversity is associated with use of systemic antibiotics and more pronounced in gastrointestinal graftversus-host disease. Biol Blood Marrow Transplantat. 2014;20(5):640-645.

24. Staffas A, Burgos da Silva M, van den Brink MR. The intestinal microbiota in allogeneic hematopoietic cell transplant and graft-versus-host disease. Blood. 2017;129(8):927 933.

25. Zeiser R, Vago L. Mechanisms of immune escape after allogeneic hematopoietic cell transplantation. Blood. 2019;133(12):12901297

26. Amaral JD, Viana RJ, Ramalho RM, Steer CI, Rodrigues CM. Bile acids: regulation of apoptosis by ursodeoxycholic acid. J Lipid Res. 2009;50(9):1721-1734

27. Rodrigues CM, Fan G, Wong PY, Kren BT, Steer CJ. Ursodeoxycholic acid may inhibit deoxycholic acid-induced apoptosis by modulating mitochondrial transmembrane potential and reactive oxygen species production. Mol Med. 1998;4(3):165-178.

28. Faubion WA, Guicciardi ME, Miyoshi $\mathrm{H}$, et al. Toxic bile salts induce rodent hepatocyte apoptosis via direct activation of Fas. J Clin Invest. 1999;103(1):137-145.

29. Yerushalmi B, Dahl R, Devereaux MW, Gumpricht E, Sokol RJ. Bile acid-induced rat hepatocyte apoptosis is inhibited by antioxidants and blockers of the mitochondrial permeability transition. Hepatology. 2001;33(3):616-626.
30. Rust C, Karnitz LM, Paya CV, Moscat J, Simari RD, Gores GJ. The bile acid taurochenodeoxycholate activates a phosphatidylinositol 3-kinase-dependent survival signaling cascade. J Biol Chem. 2000 275(26):20210-20216

31. Torchia EC, Stolz A, Agellon LB. Differential modulation of cellular death and survival pathways by conjugated bile acids. BMC Biochem. 2001;2:11.

32. Rodrigues CM, Fan G, Ma X, Kren BT, Steer CJ. A novel role for ursodeoxycholic acid in inhibiting apoptosis by modulating mitochondrial membrane perturbation. J Clin Invest. 1998;101(12):2790-2799.

33. Rodrigues CM, Ma X, Linehan-Stieers C, Fan G, Kren BT, Steer CJ. Ursodeoxycholic acid prevents cytochrome $\mathrm{c}$ release in apoptosis by inhibiting mitochondrial membrane depolarization and channel formation. Cell Death Differ. 1999;6(9):842-854.

34. Schoemaker MH, Conde de la Rosa I, Buist-Homan $M$, et al. Tauroursodeoxycholic acid protects rat hepatocytes from bile acid-induced apoptosis via activation of survival pathways. Hepatology. 2004;39(6):1563-1573.

35. Im E, Martinez JD. Ursodeoxycholic acid (UDCA) can inhibit deoxycholic acid (DCA)-induced apoptosis via modulation of EGFR/Raf-1/ERK signaling in human colon cancer cells. J Nutr. 2004;134(2):483486.

36. Vandewynckel YP, Laukens D, Devisscher $\mathrm{L}$, et al. Tauroursodeoxycholic acid dampens oncogenic apoptosis induced by endoplasmic reticulum stress during hepatocarcinogen exposure. Oncotarget. 2015 6(29):28011-28025.

37. Peng $\mathrm{P}$, Ma Q, Wang $\mathrm{L}$, et al. Preconditioning with tauroursodeoxycholic acid protects against contrast-induced $\mathrm{HK}-2$ cell apoptosis by inhibiting endoplasmic reticulum stress. Angiology. 2015; 66(10) 941-949.

38. Ruutu T, Juvonen E, Remberger M, et al. Improved survival with ursodeoxycholic acid prophylaxis in allogeneic stem cell transplantation: long-term follow-up of a randomized study. Biol Blood Marrow Transplant. 2014;20(1):135-138.

39. Essell JH, Thompson JM, Harman GS, et al. Pilot trial of prophylactic ursodiol to decrease the incidence of veno-occlusive disease of the liver in allogeneic bone marrow transplant patients. Bone Marrow Transplant. 1992;10(4):367-372.

40. Thornley I, Lehmann LE, Sung L, et al. A multiagent strategy to decrease regimenrelated toxicity in children undergoing allogeneic hematopoietic stem cell transplantation. Biol Blood Marrow Transplant. 2004 10(9):635-644.

41. Tay J, Tinmouth A, Fergusson D, Huebsch L, Allan DS. Systematic review of controlled clinical trials on the use of ursodeoxycholic acid for the prevention of hepatic veno-occlusive disease in hematopoietic stem cell transplantation. Biol Blood Marrow Transplant. 2007; 13(2):206-217.

42. Koyama M, Mukhopadhyay P, Schuster IS 
et al. MHC class II antigen presentation by the intestinal epithelium initiates graft-versus-host disease and is influenced by the microbiota. Immunity. 2019;51(5):885-898 e887.

43. Sombetzki M, Fuchs CD, Fickert P, et al. 24nor-ursodeoxycholic acid ameliorates inflammatory response and liver fibrosis in a murine model of hepatic schistosomiasis. J Hepatol. 2015;62(4):871-878.

44. Ridlon JM, Kang DJ, Hylemon PB, Bajaj JS. Bile acids and the gut microbiome. Curr Opin Gastroenterol. 2014;30(3):332-338.

45. Distrutti E, Santucci L, Cipriani S, et al. Bile acid activated receptors are targets for regulation of integrity of gastrointestinal mucosa. J Gastroenterol. 2015;50(7):707719.

46. Han S, Li T, Ellis E, Strom S, Chiang JY. A novel bile acid-activated vitamin D receptor signaling in human hepatocytes. Mol Endocrinol. 2010;24(6):1151-1164.

47. Gadaleta RM, van Erpecum KJ, Oldenburg $\mathrm{B}$, et al. Farnesoid $\mathrm{X}$ receptor activation inhibits inflammation and preserves the intestinal barrier in inflammatory bowel disease. Gut. 2011;60(4):463-472.

48. Biagioli M, Carino A, Cipriani S, et al. The bile acid receptor GPBAR1 regulates the M1/M2 phenotype of intestinal macrophages and activation of GPBAR1 rescues mice from murine colitis. Immunol. 2017;199(2):718-733.

49. Maruyama T, Miyamoto Y, Nakamura T, et al. Identification of membrane-type receptor for bile acids (M-BAR). Biochem Biophys Res Commun. 2002;298(5):714 719.

50. Duboc H, Tache Y, Hofmann AF. The bile acid TGR5 membrane receptor: from basic research to clinical application. Dig Liver Dis. 2014;46(4):302-312. 